\title{
Target mass effects in polarized virtual photon structure functions
}

\author{
Hideshi Baba, ${ }^{*}$ Ken Sasaki, ${ }^{\dagger}$ and Tsuneo Uematsu ${ }^{\ddagger}$ \\ Graduate School of Human and Environmental Studies, Kyoto University, Yoshida, Kyoto 606-8501, Japan, \\ Dept. of Physics, Faculty of Engineering, Yokohama National University, Yokohama 240-8501, Japan, \\ and Dept. of Physics, Graduate School of Science, Kyoto University, Yoshida, Kyoto 606-8501, Japan
}

(Received 11 July 2003; published 30 September 2003)

\begin{abstract}
We study target mass effects in the polarized virtual photon structure functions $g_{1}^{\gamma}\left(x, Q^{2}, P^{2}\right), g_{2}^{\gamma}\left(x, Q^{2}, P^{2}\right)$ in the kinematic region $\Lambda^{2} \ll P^{2} \ll Q^{2}$, where $-Q^{2}\left(-P^{2}\right)$ is the mass squared of the probe (target) photon. We obtain the expressions for $g_{1}^{\gamma}\left(x, Q^{2}, P^{2}\right)$ and $g_{2}^{\gamma}\left(x, Q^{2}, P^{2}\right)$ in closed form by inverting the Nachtmann moments for the twist-2 and twist-3 operators. Numerical analysis shows that target mass effects appear at large $x$ and become sizable near $x_{\max }\left[=1 /\left[1+\left(P^{2} / Q^{2}\right)\right]\right]$, the maximal value of $x$, as the ratio $P^{2} / Q^{2}$ increases. Target mass effects for the sum rules of $g_{1}^{\gamma}$ and $g_{2}^{\gamma}$ are also discussed.
\end{abstract}

DOI: 10.1103/PhysRevD.68.054025

PACS number(s): 12.38.Bx, 13.60.Hb, 13.88.+e

\section{INTRODUCTION}

The investigation of the photon structure is an active field of research both theoretically and experimentally [1-4]. In recent years, there has been growing interest in the study of the spin structure of photon. In particular, the first moment of the polarized photon structure function $g_{1}^{\gamma}$ has attracted much attention in connection with its relevance to the QED and QCD axial anomaly [5-9]. The polarized photon structure functions may be extracted from resolved photon processes in the polarized version of the DESY ep collider HERA. More directly, they can be measured from two-photon processes in the polarized $e^{+} e^{-}$collider experiments (Fig. 1), where $-Q^{2}\left(-P^{2}\right)$ is the mass squared of the probe (target) photon.

For a real photon $\left(P^{2}=0\right)$ target, there exists only one spin-dependent structure function $g_{1}^{\gamma}\left(x, Q^{2}\right)$. The QCD analysis for $g_{1}^{\gamma}$ was performed in the leading order (LO) [10] and in the next-to-leading order (NLO) $[11,12]$. In the case of a virtual photon target $\left(P^{2} \neq 0\right)$ there appear two spindependent structure functions, $g_{1}^{\gamma}\left(x, Q^{2}, P^{2}\right)$ and $g_{2}^{\gamma}\left(x, Q^{2}, P^{2}\right)$. The former has been investigated up to the NLO in QCD by the present authors in [13], and also in the second paper of [12]. In fact, we have analyzed in [13] the structure function $g_{1}^{\gamma}\left(x, Q^{2}, P^{2}\right)$ in the kinematical region $\Lambda^{2} \ll P^{2} \ll Q^{2}$, where $\Lambda$ is the QCD scale parameter. The advantage of studying a virtual photon target in that kinematical region is that we can calculate structure functions entirely up to the NLO by the perturbative method [14], which is contrasted with the case of the real photon target where in the NLO there exist nonperturbative pieces. As for the structure function $g_{2}^{\gamma}\left(x, Q^{2}, P^{2}\right)$, the analysis has not made much progress owing to the difficulty arising from the relevant twist-3 operators. So far only the LO QCD corrections to the flavor nonsinglet part of $g_{2}^{\gamma}$ have been calculated in the large $N_{c}$ limit [15].

\footnotetext{
*Email address: baba@phys.h.kyoto-u.ac.jp

†Email address: sasaki@phys.ynu.ac.jp

Email address: uematsu@ phys.h.kyoto-u.ac.jp
}

If the target is real photon $\left(P^{2}=0\right)$, there is no need to consider target mass corrections. But when the target becomes off-shell, for example, $P^{2} \geqslant M^{2}$, where $M$ is the nucleon mass, and for relatively low values of $Q^{2}$, contributions suppressed by powers of $P^{2} / Q^{2}$ may become important. Then we need to take into account these target mass contributions just like the case of the nucleon structure functions. The consideration of target mass effects (TME) is important by another reason. For the virtual photon target, the maximal value of the Bjorken variable $x$ is not 1 but

$$
x_{\max }=\frac{1}{1+\frac{P^{2}}{Q^{2}}},
$$

due to the constraint $(p+q)^{2} \geqslant 0$, which is contrasted with the nucleon case where $x_{\max }=1$. The structure functions

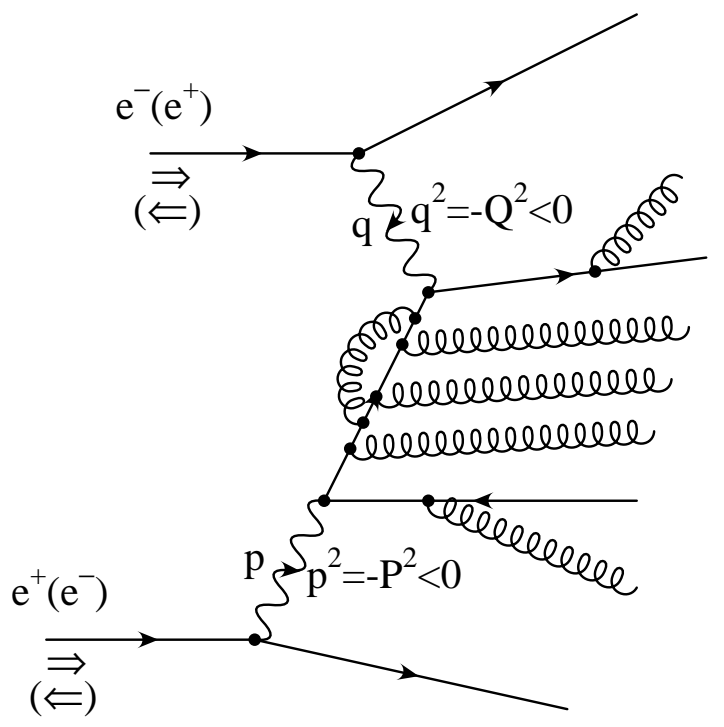

FIG. 1. Deep inelastic scattering on a polarized virtual photon in a polarized $e^{+} e^{-}$collision, $e^{+} e^{-} \rightarrow e^{+} e^{-}+$hadrons (quarks and gluons). The arrows indicate the polarizations of the $e^{+}$and $e^{-}$. The mass squared of the "probe" ("target") photon is $-Q^{2}\left(-P^{2}\right)\left(\Lambda^{2} \ll P^{2} \ll Q^{2}\right)$ with $\Lambda$ being the QCD scale parameter. 
should vanish at $x=x_{\max }$. However, the NLO QCD result [13] for $g_{1}^{\gamma}\left(x, Q^{2}, P^{2}\right)$ shows that the predicted graph does not vanish but remains finite at $x=x_{\max }$. This flaw is coming from the fact that TME have not been taken into account in the analysis. The target mass corrections have been studied in the past for the cases of unpolarized $[16,17]$ and polarized [18-22] nucleon structure functions.

In this paper we investigate TME in the polarized virtual photon structure functions $g_{1}^{\gamma}\left(x, Q^{2}, P^{2}\right)$ and $g_{2}^{\gamma}\left(x, Q^{2}, P^{2}\right)$. In the analysis of $g_{1}^{\gamma}$ in [13], the formalism of the operator product expansion (OPE) supplemented by the renormalization group method was used. The photon matrix elements of the relevant traceless operators in the OPE are expressed by traceless tensors. These tensors contain many trace terms so that they satisfy the tracelessness conditions. The basic idea for computing the target mass corrections exactly is to take account of trace terms in the traceless tensors properly. There are two methods used so far for collecting all those trace terms. One, which was introduced by Nachtmann [16], is to make use of Gegenbauer polynomials to express the contractions between $q_{\mu_{1}} \cdots q_{\mu_{n-1}}$ and the traceless tensors [6,1820]. This method leads to the Nachtmann moments for the twist- 2 and twist- 3 operators with definite spin. The other, first used by Georgi and Politzer [17], is to write traceless tensors explicitly and then to collect trace terms and sum them up. Through the latter approach, the moments of structure functions are expressed as functions of the reduced operator matrix elements and coefficient functions with different spins. Actually both methods give equivalent results. In this paper we apply the former method to study target mass corrections to the structure functions $g_{1}^{\gamma}$ and $g_{2}^{\gamma}$.

In the next section we discuss the framework for analyzing the TME based on the OPE and derive the Nachtmann moments for the twist- 2 and twist- 3 operators with definite spin using the orthogonality relations of Gegenbauer polynomials. In Sec. III, by inverting the Nachtmann moments, we obtain the explicit expressions for the polarized photon structure functions $g_{1}^{\gamma}\left(x, Q^{2}, P^{2}\right)$ and $g_{2}^{\gamma}\left(x, Q^{2}, P^{2}\right)$ with TME included. In Sec. IV we perform the numerical analysis and show that target mass corrections become sizable near $x_{\max }$. Section V is devoted to the conclusion.

\section{NACHTMANN MOMENTS}

Let us consider the virtual photon-photon forward scattering for $\gamma(q)+\gamma(p) \rightarrow \gamma(q)+\gamma(p)$ illustrated in Fig. 2,

$$
\begin{aligned}
T_{\mu \nu \rho \tau}(p, q)= & i \int d^{4} x d^{4} y d^{4} z e^{i q \cdot x} e^{i p \cdot(y-z)} \\
& \times\left\langle 0\left|T\left[J_{\mu}(x) J_{\nu}(0) J_{\rho}(y) J_{\tau}(z)\right]\right| 0\right\rangle,
\end{aligned}
$$

where $J$ is the electromagnetic current, and $q$ and $p$ are the four-momenta of two photons. Its absorptive part is related to the structure tensor $W_{\mu \nu \rho \tau}(p, q)$ for the target photon with mass squared $p^{2}=-P^{2}$ probed by the photon with $q^{2}=$ $-Q^{2}$ :

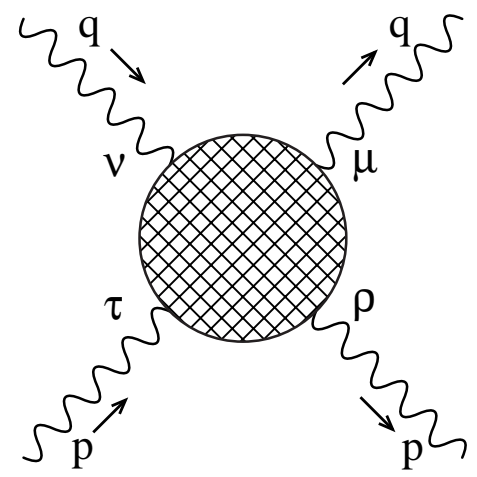

FIG. 2. Forward scattering of a virtual photon with momentum $q$ and another virtual photon with momentum $p$. The Lorentz indices are denoted by $\mu, \nu, \rho, \tau$.

$$
W_{\mu \nu \rho \tau}(p, q)=\frac{1}{\pi} \operatorname{Im} T_{\mu \nu \rho \tau}(p, q)
$$

The antisymmetric part $W_{\mu \nu \rho \tau}^{A}$ under the interchange $\mu \leftrightarrow \nu$ and $\rho \leftrightarrow \tau$ can be decomposed as

$$
\begin{aligned}
W_{\mu \nu \rho \tau}^{A}= & \epsilon_{\mu \nu \lambda \sigma} q^{\lambda} \epsilon_{\rho \tau}{ }^{\sigma \beta} p_{\beta} \frac{1}{p \cdot q} g_{1}^{\gamma}+\epsilon_{\mu \nu \lambda \sigma} q^{\lambda}\left(p \cdot q \epsilon_{\rho \tau}{ }^{\sigma \beta} p_{\beta}\right. \\
& \left.-\epsilon_{\rho \tau \alpha \beta} p^{\beta} p^{\sigma} q^{\alpha}\right) \frac{1}{(p \cdot q)^{2}} g_{2}^{\gamma},
\end{aligned}
$$

which gives two spin-dependent structure functions, $g_{1}^{\gamma}\left(x, Q^{2}, P^{2}\right)$ and $g_{2}^{\gamma}\left(x, Q^{2}, P^{2}\right)$. When the target is real photon $\left(P^{2}=0\right), g_{2}^{\gamma}$ is identically zero, and there exists only one spin structure function, $g_{1}^{\gamma}\left(x, Q^{2}\right)$. On the other hand, for the off-shell or virtual photon $\left(P^{2} \neq 0\right)$ target, we have two spindependent structure functions $g_{1}^{\gamma}$ and $g_{2}^{\gamma}$.

For the analysis of spin structure functions, we apply the OPE for the product of two electromagnetic currents. We obtain for the $\mu-\nu$ antisymmetric part

$$
\begin{aligned}
i \int d^{4} x & e^{i q \cdot x} T\left[J_{\mu}(x) J_{\nu}(0)\right]^{A} \\
= & -i \epsilon_{\mu \nu \lambda \sigma} q^{\lambda} \sum_{n=1,3, \ldots}\left(\frac{2}{Q^{2}}\right)^{n} q_{\mu_{1}} \cdots q_{\mu_{n-1}} \\
& \times\left\{\sum_{i} E_{(2) i}^{n} R_{(2) i}^{\sigma \mu_{1} \cdots \mu_{n-1}}+\sum_{i} E_{(3) i}^{n} R_{(3) i}^{\sigma \mu_{1} \cdots \mu_{n-1}}\right\},
\end{aligned}
$$

where $R_{(2) i}^{n}$ and $R_{(3) i}^{n}$ are the twist-2 and twist-3 operators, respectively, and are both traceless, and $E_{(2) i}^{n}$ and $E_{(3) i}^{n}$ are corresponding coefficient functions. The twist-2 operators $R_{(2) i}^{n}$ have totally symmetric Lorentz indices $\sigma \mu_{1} \cdots \mu_{n-1}$, while the indices of twist-3 operators $R_{(3) i}^{n}$ are totally symmetric among $\mu_{1} \cdots \mu_{n-1}$ but antisymmetric under $\sigma \leftrightarrow \mu_{i}$. 
In the case of photon target we evaluate "matrix elements" of the traceless operators $R_{(2) i}^{n}$ and $R_{(3) i}^{n}$ sandwiched by two photon states with momentum $p$, which are written in the following forms:

$$
\begin{aligned}
\langle 0| & T\left[A_{\rho}(-p) R_{(2) i}^{\sigma \mu_{1} \cdots \mu_{n-1}} A_{\tau}(p)\right]|0\rangle_{\mathrm{Amp}} \\
& =-i a_{(2) i}^{\gamma, n} M_{(2) \rho \tau}^{\sigma \mu_{1} \cdots \mu_{n-1}}, \\
\langle 0| & T\left[A_{\rho}(-p) R_{(3) i}^{\sigma \mu_{1} \cdots \mu_{n-1}} A_{\tau}(p)\right]|0\rangle_{\mathrm{Amp}} \\
& =-i a_{(3) i}^{\gamma, n} M_{(3) \rho \tau}^{\left[\sigma,\left\{\mu_{1}\right] \cdots \mu_{n-1}\right\}},
\end{aligned}
$$

where the subscript "Amp" stands for the amputation of external photon lines, and $a_{(2) i}^{\gamma, n}$ and $a_{(3) i}^{\gamma, n}$ are reduced photon matrix elements. The tensors $M_{(2) \rho \tau}^{\sigma \mu_{1} \cdots \mu_{n-1}}$ and $M_{(3) \rho \tau}^{\left[\sigma,\left\{\mu_{1}\right] \cdots \mu_{n-1}\right\}}$ are given by

$$
\begin{aligned}
& M_{(2) \rho \tau}^{\sigma \mu_{1} \cdots \mu_{n-1}} \equiv \frac{1}{n}\left[\epsilon_{\rho \tau \alpha}{ }^{\sigma} p^{\mu_{1} \cdots} p^{\mu_{n-1}}\right. \\
& \left.+\sum_{j=1}^{n-1} p^{\sigma} p^{\mu_{1} \cdots \epsilon_{\rho \tau \alpha}}{ }^{\mu_{j}} \cdots p^{\mu_{n-1}}\right] p^{\alpha} \\
& \text {-(trace terms }) \text {, } \\
& M_{(3) \rho \tau}^{\left[\sigma,\left\{\mu_{1}\right] \cdots \mu_{n-1}\right\}} \equiv\left[\frac{n-1}{n} \epsilon_{\rho \tau \alpha}{ }^{\sigma} p^{\mu_{1} \cdots} p^{\mu_{n-1}}\right. \\
& \left.-\frac{1}{n} \sum_{j=1}^{n-1} p^{\sigma} p^{\mu_{1}} \cdots \epsilon_{\rho \tau \alpha} \mu_{j} \ldots p^{\mu_{n-1}}\right] p^{\alpha} \\
& \text { - (trace terms), }
\end{aligned}
$$

and satisfy the traceless conditions,

$$
g_{\sigma \mu_{i}} M_{(k) \rho \tau}^{\sigma \mu_{1} \cdots \mu_{n-1}}=0, \quad g_{\mu_{i} \mu_{j}} M_{(k) \rho \tau}^{\sigma \mu_{1} \cdots \mu_{n-1}}=0 \quad(k=2,3) .
$$

Taking the "matrix elements" of (2.4) with the virtual photon states, we obtain for the deep-inelastic photon-photon forward scattering amplitude

$$
\begin{aligned}
T_{\mu \nu \rho \tau}^{A}= & i \int d^{4} x e^{i q \cdot x}\langle 0| T\left\{A_{\rho}(-p)\right. \\
& \left.\times\left[J_{\mu}(x) J_{\nu}(0)\right]^{A} A_{\tau}(p)\right\}|0\rangle_{\mathrm{Amp}} \\
= & -\epsilon_{\mu \nu \lambda \sigma} q^{\lambda} \sum_{n=1,3, \ldots}\left(\frac{2}{Q^{2}}\right)^{n} q_{\mu_{1}} \cdots q_{\mu_{n-1}} \\
& \times\left\{\sum_{i} a_{(2) i}^{\gamma, n} E_{(2) i}^{n} M_{(2) \rho \tau}^{\sigma \mu_{1} \cdots \mu_{n-1}}\right. \\
& \left.+a_{(3) i}^{\gamma, n} E_{(3) i}^{n} M_{(3) \rho \tau}^{\left[\sigma,\left\{\mu_{1}\right] \cdots \mu_{n-1}\right\}}\right\} .
\end{aligned}
$$

The basic idea for treating target mass corrections exactly is to take account of trace terms in the traceless tensors prop- erly. We evaluate the contraction between $q_{\mu_{1}} \cdots q_{\mu_{n-1}}$ and the traceless tensors without neglecting any of the trace terms in Eqs. (2.7) and (2.8). The results are expressed in terms of Gegenbauer polynomials $[16,18,19]$. Denoting $M_{(2) \rho \tau}^{\sigma \mu_{1} \cdots \mu_{n-1}} \equiv \tilde{M}_{(2) \beta}^{\sigma \mu_{1} \cdots \mu_{n-1}} \epsilon_{\rho \tau \alpha}{ }^{\beta} p^{\alpha} \quad$ and $\quad M_{(3) \rho \tau}^{\left[\sigma,\left\{\mu_{1}\right] \cdots \mu_{n-1}\right\}}$

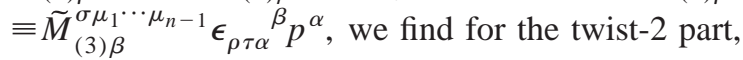

$$
\begin{aligned}
q_{\mu_{1}} \cdots q_{\mu_{n-1}} \tilde{M}_{(2) \beta}^{\sigma \mu_{1} \cdots \mu_{n-1}=} & \frac{1}{n^{2}}\left[\delta_{\beta}^{\sigma} a^{n-1} C_{n-1}^{(2)}(\eta)\right. \\
& \left.+q_{\beta} p^{\sigma} a^{n-2} 2 C_{n-2}^{(3)}(\eta)\right] \\
& +\left(\text { terms with } p_{\beta} \text { or } q^{\sigma}\right)
\end{aligned}
$$

and for the twist-3 part

$$
\begin{aligned}
q_{\mu_{1}} \cdots & q_{\mu_{n-1}} \tilde{M}_{(3) \beta}^{\sigma \mu_{1} \cdots \mu_{n-1}} \\
= & \delta_{\beta} \frac{a^{n-1}}{n^{2}}\left[(n-1) C_{n-1}^{(2)}(\eta)-(n+1) C_{n-3}^{(2)}(\eta)\right] \\
& \quad-q_{\beta} p^{\sigma} \frac{2 a^{n-2}}{n^{2}}\left[C_{n-2}^{(3)}(\eta)+C_{n-4}^{(3)}(\eta)\right] \\
& +\left(\text { terms with } p_{\beta} \text { or } q^{\sigma}\right)
\end{aligned}
$$

where $a=-\frac{1}{2} P Q, \eta=-p \cdot q / P Q$ and $C_{n}^{(\nu)}(\eta)$ 's are Gegenbauer polynomials (see Appendix A). In fact in the above two equations there appear terms with $p_{\beta}$ or $q^{\sigma}$. These terms give null results when they are multiplied by $\epsilon_{\rho \tau \alpha}{ }^{\beta} p^{\alpha}$ and $\epsilon_{\mu \nu \lambda \sigma} q^{\lambda}$. [See Appendix B for the derivation of Eqs. (2.11) and (2.12).]

We decompose the amplitude $T_{\mu \nu \rho \tau}^{A}$ as

$$
\begin{aligned}
T_{\mu \nu \rho \tau}^{A}= & \epsilon_{\mu \nu \lambda \sigma} q^{\lambda} \epsilon_{\rho \tau}{ }^{\sigma \beta} p_{\beta} \frac{1}{p \cdot q}\left(v_{1}^{\gamma}+v_{2}^{\gamma}\right) \\
& -\epsilon_{\mu \nu \lambda \sigma} q^{\lambda} p^{\sigma} \epsilon_{\rho \tau \alpha \beta} q^{\alpha} p^{\beta} \frac{1}{(p \cdot q)^{2}} v_{2}^{\gamma},
\end{aligned}
$$

then, using the above results on the contractions we find

$$
\begin{aligned}
v_{1}^{\gamma}+v_{2}^{\gamma}= & \sum_{n=1,3, \ldots} \sum_{i} a_{(2) i}^{\gamma, n} E_{(2) i}^{n}\left(-\frac{P}{Q}\right)^{n} \frac{1}{n^{2}} 2 \eta C_{n-1}^{(2)}(\eta) \\
& -\sum_{n=3,5, \ldots} \sum_{i} a_{(3) i}^{\gamma, n} E_{(3) i}^{n}\left(-\frac{P}{Q}\right)^{n} \frac{1}{n^{2}} 2 \eta \\
& \times\left[(n+1) C_{n-3}^{(2)}(\eta)-(n-1) C_{n-1}^{(2)}(\eta)\right] \\
v_{2}^{\gamma}= & -\sum_{n=1,3, \ldots} \sum_{i} a_{(2) i}^{\gamma, n} E_{(2) i}^{n}\left(-\frac{P}{Q}\right)^{n} \frac{1}{n^{2}} 8 \eta^{2} C_{n-2}^{(3)}(\eta) \\
& +\sum_{n=3,5, \ldots} \sum_{i} a_{(3) i}^{\gamma, n} E_{(3) i}^{n}\left(-\frac{P}{Q}\right)^{n} \frac{1}{n^{2}} 8 \eta^{2}\left[C_{n-2}^{(3)}(\eta)\right. \\
& \left.+C_{n-4}^{(3)}(\eta)\right] .
\end{aligned}
$$


Here we compare the expressions of $v_{1}^{\gamma}+v_{2}^{\gamma}$ and $v_{2}^{\gamma}$ with those given in Eqs. (8) and (9) of Ref. [19], which are the invariant amplitudes for polarized deep inelastic leptonnucleon scattering with target nucleon mass corrections being taken into account. Apart from the reduced matrix elements and coefficient functions, the expressions for both photon and nucleon targets are exactly the same once the replacement of $M$ with $-i P$, or vice versa, is made. This is due to the fact that the factor $\left[\epsilon_{\rho \tau \alpha}{ }^{\sigma} p^{\alpha}\right]\left(\left[\epsilon_{\rho \tau \alpha}{ }^{\mu j} p^{\alpha}\right]\right)$ appearing in the photon matrix elements [Eqs. (2.7) and (2.8)] and in the decomposition [Eq. (2.13)] plays the same role as nucleon spin $s^{\sigma}\left(s^{\mu_{j}}\right)$, since $p_{\sigma} \epsilon_{\rho \tau \alpha}{ }^{\sigma} p^{\alpha}=0 \quad\left(p_{\mu_{j}} \epsilon_{\rho \tau \alpha}{ }^{\mu_{j}} p^{\alpha}\right.$ $=0$ ). Thus the tensor structures of both polarized photon and polarized nucleon matrix elements are exactly the same. The only difference between the two is that $p^{2}=-P^{2}$ for photon target and $p^{2}=M^{2}$ for nucleon.

Now we follow the same procedures as were taken by Wandzura [18] and in Ref. [19] for the polarized nucleon case, and we obtain the analytic expression of the Nachtmann moments for the twist- 2 and twist- 3 operators with definite spin $n$. First we write the dispersion relations for $v_{1}^{\gamma}$ and $v_{2}^{\gamma}$ and denote

$$
g_{1,2}^{\gamma}=\frac{1}{\pi} \operatorname{Im} v_{1,2}^{\gamma} .
$$

Second, using the orthogonality relations [Eq. (A8)] and an integration formula [Eq. (A9)] for Gegenbauer polynomials $C_{n}^{(\nu)}(\eta)$, we project out $\Sigma_{i} a_{(2) i}^{\gamma, n} E_{(2) i}^{n}$ and $\Sigma_{i} a_{(3) i}^{\gamma, n} E_{(3) i}^{n}$ with definite spin $n$, which still include the infinite series in powers of $P^{2} / Q^{2}$. Third, we sum up those infinite series and express them in compact analytic forms [19]. Then we obtain

$$
\begin{aligned}
M_{2}^{n} \equiv & \sum_{i} a_{(2) i}^{\gamma, n} E_{(2) i}^{n}\left(Q^{2}, P^{2}, g\right) \\
= & \int_{0}^{x_{\max }} \frac{d x}{x^{2}} \xi^{n+1}\left[\left\{\frac{x}{\xi}+\frac{n^{2}}{(n+2)^{2}} \frac{P^{2} x \xi}{Q^{2}}\right\} g_{1}^{\gamma}\left(x, Q^{2}, P^{2}\right)\right. \\
& \left.+\frac{4 n}{n+2} \frac{P^{2} x^{2}}{Q^{2}} g_{2}^{\gamma}\left(x, Q^{2}, P^{2}\right)\right] \quad(n=1,3, \ldots), \quad(2.17) \\
M_{3}^{n} \equiv & \sum_{i} a_{(3) i}^{\gamma, n} E_{(3) i}^{n}\left(Q^{2}, P^{2}, g\right) \\
= & \int_{0}^{x_{\max }} \frac{d x}{x^{2}} \xi^{n+1}\left[\frac{x}{\xi} g_{1}^{\gamma}\left(x, Q^{2}, P^{2}\right)+\left\{\frac{n}{n-1} \frac{x^{2}}{\xi^{2}}\right.\right. \\
& \left.\left.+\frac{n}{n+1} \frac{P^{2} x^{2}}{Q^{2}}\right\} g_{2}^{\gamma}\left(x, Q^{2}, P^{2}\right)\right] \quad(n=3,5, \ldots),
\end{aligned}
$$

where $x=Q^{2} /(2 p \cdot q)$ and $\xi$, the so-called $\xi$-scaling variable, is given by

$$
\xi=\frac{2 x}{1+\sqrt{1-\frac{4 P^{2} x^{2}}{Q^{2}}}} .
$$

In fact, the above results for the Nachtmann moments are reproduced from the counterparts in the case of spindependent nucleon structure functions, Eqs. (18) and (19) of Ref. [19], by replacing the target nucleon mass $M$ with $-i P$.

We see from Eq. (1.1) that the maximal value of $x$ is not 1 but $1 /\left[1+\left(P^{2} / Q^{2}\right)\right]$. Therefore, the allowed range of $\xi$ is $0 \leqslant \xi \leqslant 1$. It is important to note that $\xi\left(x_{\max }\right)=1$ for the virtual photon target. In the nucleon case, however, the constraint $(p+q)^{2} \geqslant M^{2}$ gives $x_{\max }=1$. Changing $P^{2}$ in Eq. (2.19) to $-M^{2}$, we get that $\xi(x=1)<1$. This leads to a well-known difficulty at $x=1$ in the analysis of target mass corrections to nucleon structure functions, both in unpolarized and polarized cases. The nucleon structure functions should vanish at $x=1$ kinematically, while their expressions, once target mass effects are taken into account, vanish at $\xi$ $=1$ but remain non-zero when $\xi(x=1)<1$. The resolution to this problem was argued in Refs. [21-23] by considering the dynamical higher-twist effects. On the other hand, in the case of a virtual photon target, we have no such difficulty. When $Q^{2}, P^{2} \gg \Lambda^{2}$, we can put the constraint as $(p+q)^{2}$ $\geqslant 0$ and this leads to $x_{\max }$ given in Eq. (1.1). We will see later that the virtual photon structure functions with target mass corrections included do vanish at $x_{\max }$, since $\xi\left(x_{\max }\right)$ $=1$.

The left-hand side of Eqs. (2.17) and (2.18), $M_{2}^{n}$ and $M_{3}^{n}$, can be computed in perturbative QCD up to NLO, since in the kinematical region $Q^{2} \gg P^{2} \gg \Lambda^{2}$ both the reduced photon matrix elements $a_{(k) i}^{\gamma, n}(k=2,3)$ and coefficient functions $E_{(k) i}^{n}\left(Q^{2}, P^{2}, g\right)(k=2,3)$ are calculable. In fact, the perturbative QCD calculation of $M_{2}^{n}$ has been done in LO [10] and in NLO [13], while the QCD analysis of $M_{3}^{n}$ has been carried out in LO for the flavor non-singlet part in the limit of large $N_{c}$ [15]. Once the moments $M_{2}^{n}$ and $M_{3}^{n}$ are known, we can derive $g_{1}^{\gamma}\left(x, Q^{2}, P^{2}\right)$ and $g_{2}^{\gamma}\left(x, Q^{2}, P^{2}\right)$ as functions of $x$ by inverting $M_{2}^{n}$ and $M_{3}^{n}$, which will be discussed in the next section.

\section{INVERTING THE MOMENTS}

First let us rewrite the Nachtmann moments in the variable $\xi$ and we get

$$
\begin{aligned}
M_{2}^{n}= & \int_{0}^{1} d \xi \xi^{n-1}\left[\left\{1+\frac{n^{2}}{(n+2)^{2}} \kappa \xi^{2}\right\} \frac{1-\kappa \xi^{2}}{1+\kappa \xi^{2}} g_{1}^{\gamma}\left(x, Q^{2}, P^{2}\right)\right. \\
& \left.+\frac{n}{n+2} 4 \kappa \xi^{2} \frac{1-\kappa \xi^{2}}{\left(1+\kappa \xi^{2}\right)^{2}} g_{2}^{\gamma}\left(x, Q^{2}, P^{2}\right)\right] \\
M_{3}^{n}= & \int_{0}^{1} d \xi \xi^{n-1}\left[\frac{1-\kappa \xi^{2}}{1+\kappa \xi^{2}} g_{1}^{\gamma}\left(x, Q^{2}, P^{2}\right)\right. \\
& \left.+\left\{\frac{n}{n-1}+\frac{n}{n+1} \kappa \xi^{2}\right\} \frac{1-\kappa \xi^{2}}{\left(1+\kappa \xi^{2}\right)^{2}} g_{2}^{\gamma}\left(x, Q^{2}, P^{2}\right)\right],
\end{aligned}
$$


where we have put $\kappa \equiv P^{2} / Q^{2}$. We define

$$
\begin{aligned}
& A(\xi) \equiv \frac{1-\kappa \xi^{2}}{1+\kappa \xi^{2}} g_{1}^{\gamma}\left(x, Q^{2}, P^{2}\right), \\
& B(\xi) \equiv \frac{1-\kappa \xi^{2}}{\left(1+\kappa \xi^{2}\right)^{2}} g_{2}^{\gamma}\left(x, Q^{2}, P^{2}\right) .
\end{aligned}
$$

The boundary conditions for $A(\xi)$ and $B(\xi)$ are $A(\xi=1)$ $=B(\xi=1)=0$, since $g_{1}^{\gamma}\left(x_{\max }, Q^{2}, P^{2}\right)=g_{2}^{\gamma}\left(x_{\max }, Q^{2}, P^{2}\right)=0$ and $\xi\left(x_{\max }\right)=1$. Now introducing the following four functions:

$$
\begin{aligned}
\widetilde{A}(\xi) & =\int_{\xi}^{1} \frac{d \xi^{\prime}}{\xi^{\prime}} \int_{\xi^{\prime}}^{1} \frac{d \xi^{\prime \prime}}{\xi^{\prime \prime}} A\left(\xi^{\prime \prime}\right), \\
B_{-1}(\xi) & =\int_{\xi}^{1} \frac{d \xi^{\prime}}{\xi^{\prime}} B\left(\xi^{\prime}\right), \\
B_{0}(\xi) & =\int_{\xi}^{1} d \xi^{\prime} B\left(\xi^{\prime}\right), \\
B_{1}(\xi) & =\int_{\xi}^{1} d \xi^{\prime}\left\{\xi^{\prime} B\left(\xi^{\prime}\right)\right\}
\end{aligned}
$$

and by partial integration we find that the above two moments are written as

$$
\begin{aligned}
\frac{M_{n}^{2}}{n^{2}}= & \int_{0}^{1} d \xi \xi^{n-1}\left[\left(1+\kappa \xi^{2}\right) \widetilde{A}(\xi)+2 \kappa\left\{B_{1}(\xi)-\xi^{2} B_{-1}(\xi)\right\}\right], \\
\frac{M_{3}^{n}}{n^{2}}= & \int_{0}^{1} d \xi \xi^{n-1}\left[\widetilde{A}(\xi)+\frac{1}{\xi}\left(1-\kappa \xi^{2}\right) B_{0}(\xi)-B_{-1}(\xi)\right. \\
& \left.+\kappa B_{1}(\xi)\right] .
\end{aligned}
$$

Inverting the moments we get

$$
\begin{aligned}
H_{a}(\xi) & =\frac{1}{2 \pi i} \int_{c-i \infty}^{c+i \infty} d n \xi^{-n}\left\{\frac{M_{2}^{n}}{n^{2}}\right\} \\
& =\left(1+\kappa \xi^{2}\right) \widetilde{A}(\xi)+2 \kappa\left\{B_{1}(\xi)-\xi^{2} B_{-1}(\xi)\right\} \\
H_{d}(\xi) & =\frac{1}{2 \pi i} \int_{c-i \infty}^{c+i \infty} d n \xi^{-n}\left\{\frac{M_{3}^{n}}{n^{2}}\right\} \\
& =\widetilde{A}(\xi)+\frac{1}{\xi}\left(1-\kappa \xi^{2}\right) B_{0}(\xi)-B_{-1}(\xi)+\kappa B_{1}(\xi),
\end{aligned}
$$

where we have adopted the notation used in Ref. [21] for $H_{a}(\xi)$ and $H_{d}(\xi)$. Further introducing the following functions [21]:

$$
G_{a, d}(\xi)=-\xi \frac{d H_{a, d}(\xi)}{d \xi}, \quad F_{a, d}(\xi)=-\frac{d G_{a, d}(\xi)}{d \xi},
$$

we differentiate both sides of Eqs. (3.8) and (3.9) by $\xi$ and get the relations between $\widetilde{A}(\xi), B_{-1}(\xi), B_{0}(\xi), B_{1}(\xi)$ and $H_{a, d}(\xi), G_{a, d}(\xi), F_{a, d}(\xi)$. Now replacing the former functions with the latter, we solve for $g_{1}^{\gamma}$ and $g_{2}^{\gamma}$ and obtain

$$
\begin{aligned}
g_{1}^{\gamma}\left(x, Q^{2}, P^{2}\right) & \\
= & 4 \kappa \xi^{2} \frac{\left(1+\kappa \xi^{2}\right)^{3}}{\left(1-\kappa \xi^{2}\right)^{5}}\left\{1+\frac{2 \kappa \xi^{2}}{\left(1+\kappa \xi^{2}\right)^{2}}\right\} H_{a}(\xi) \\
& -4 \kappa \xi^{2} \frac{\left(1+\kappa \xi^{2}\right)^{2}}{\left(1-\kappa \xi^{2}\right)^{4}}\left\{1+\frac{1}{1+\kappa \xi^{2}}\right\} G_{a}(\xi) \\
& +\xi \frac{\left(1+\kappa \xi^{2}\right)^{2}}{\left(1-\kappa \xi^{2}\right)^{3}} F_{a}(\xi)-8 \kappa \xi^{2} \frac{\left(1+\kappa \xi^{2}\right)^{3}}{\left(1-\kappa \xi^{2}\right)^{5}} \\
& \times\left\{1+\frac{2 \kappa \xi^{2}}{\left(1+\kappa \xi^{2}\right)^{2}}\right\} H_{d}(\xi)+12 \kappa \xi^{2} \frac{\left(1+\kappa \xi^{2}\right)^{2}}{\left(1-\kappa \xi^{2}\right)^{4}} G_{d}(\xi) \\
& -4 \kappa \xi^{3} \frac{1+\kappa \xi^{2}}{\left(1-\kappa \xi^{2}\right)^{3}} F_{d}(\xi), \\
g_{2}^{\gamma}(x, & \left.Q^{2}, P^{2}\right) \\
= & -6 \kappa \xi^{2} \frac{\left(1+\kappa \xi^{2}\right)^{3}}{\left(1-\kappa \xi^{2}\right)^{5}} H_{a}(\xi)+\frac{\left(1+\kappa \xi^{2}\right)^{3}}{\left(1-\kappa \xi^{2}\right)^{4}} \\
& \times\left\{1+\frac{4 \kappa \xi^{2}}{1+\kappa \xi^{2}}\right\} G_{a}(\xi)-\xi \frac{\left(1+\kappa \xi^{2}\right)^{2}}{\left(1-\kappa \xi^{2}\right)^{3}} F_{a}(\xi) \\
& +12 \kappa \xi^{2} \frac{\left(1+\kappa \xi^{2}\right)^{3}}{\left(1-\kappa \xi^{2}\right)^{5}} H_{d}(\xi)-\frac{\left(1+\kappa \xi^{2}\right)^{4}}{\left(1-\kappa \xi^{2}\right)^{4}} \\
& \times\left\{1+\frac{8 \kappa \xi^{2}}{\left(1+\kappa \xi^{2}\right)^{2}}\right\} G_{d}(\xi)+\xi \frac{\left(1+\kappa \xi^{2}\right)^{3}}{\left(1-\kappa \xi^{2}\right)^{3}} F_{d}(\xi) \\
&
\end{aligned}
$$

Equations (3.11) and (3.12) are the final formulas for the polarized photon structure functions $g_{1}^{\gamma}$ and $g_{2}^{\gamma}$ when target mass effects are taken into account. The parameter $\kappa$ represents the target mass corrections. Once the reduced photon matrix elements and coefficient functions corresponding to the relevant twist-2 and -3 operators, more specifically, $\sum_{i} a_{(2) i}^{\gamma, n} E_{(2) i}^{n}\left(Q^{2}, P^{2}, g\right)$ and $\sum_{i} a_{(3) i}^{\gamma, n} E_{(3) i}^{n}\left(Q^{2}, P^{2}, g\right)$ in Eqs. (2.17) and (2.18), are given, then we can calculate $H_{a, d}(\xi)$, $G_{a, d}(\xi)$, and $F_{a, d}(\xi)$ through Eqs. (3.8)-(3.10), and predict whole structure functions with target mass corrections. Note that by definition the functions $H_{a, d}(\xi), G_{a, d}(\xi)$, and $F_{a, d}(\xi)$ contain the logarithmic QCD corrections depending on $\ln \left(Q^{2} / \Lambda^{2}\right)$ and $\ln \left(P^{2} / \Lambda^{2}\right)$. When we set $\kappa=0$ in Eqs. (3.11) and (3.12) and Eq. (2.19), we obtain

$$
\left.g_{1}^{\gamma}\left(x, Q^{2}, P^{2}\right)\right|_{0}=x F_{a}(x),
$$




$$
\left.g_{2}^{\gamma}\left(x, Q^{2}, P^{2}\right)\right|_{0}=G_{a}(x)-x F_{a}(x)-G_{d}(x)+x F_{d}(x)
$$

for the polarized photon structure functions without target mass effects, which have been investigated in the literature $[12,13,15]$. We have suppressed the logarithmic $Q^{2}$ and $P^{2}$ dependence in the arguments of $G_{a, d}(x)$ and $F_{a, d}(x)$.

Before we proceed to numerical analysis for target mass effects on the polarized photon structure functions, let us consider the power series expansion of target mass effects. In the phenomenological analysis of target mass effects on the polarized nucleon structure functions [21], the expansion in powers of $P^{2} / Q^{2}$ was carried out and the first order terms were kept to analyze the experimental data. It would be interesting to see how good the first order approximation is in the case of the virtual photon target. We take the $x$ moments of the structure functions $g_{1,2}^{\gamma}\left(x, Q^{2}, P^{2}\right)$,

$$
\begin{aligned}
g_{1,2}^{\gamma, n} & \equiv \int_{0}^{x_{\max }} d x x^{n-1} g_{1,2}^{\gamma}\left(x, Q^{2}, P^{2}\right) \\
& =\int_{0}^{1} d \xi \frac{1-\kappa \xi^{2}}{\left(1+\kappa \xi^{2}\right)^{2}}\left(\frac{\xi}{1+\kappa \xi^{2}}\right)^{n-1} g_{1,2}^{\gamma}\left(x, Q^{2}, P^{2}\right) .
\end{aligned}
$$

Using the expressions given in Eqs. (3.11) and (3.12) for $g_{1,2}^{\gamma}\left(x, Q^{2}, P^{2}\right)$, we expand the integrands to the first order in $\kappa$. Then we obtain

$$
g_{1}^{\gamma, n}=M_{2}^{n}-\kappa \frac{n^{2}(n+1)}{(n+2)^{2}} M_{2}^{n+2}-\kappa \frac{4 n(n+1)}{(n+2)^{2}} M_{3}^{n+2}+O\left(\kappa^{2}\right),
$$

$$
\begin{aligned}
g_{2}^{\gamma, n}= & -\frac{n-1}{n} M_{2}^{n}+\frac{n-1}{n} M_{3}^{n}+\kappa \frac{n(n+1)(n-1)}{(n+2)^{2}} M_{2}^{n+2} \\
& -\kappa \frac{n^{2}(n-1)}{(n+2)^{2}} M_{3}^{n+2}+O\left(\kappa^{2}\right),
\end{aligned}
$$

where we have used the formulas

$$
\begin{array}{r}
\int_{0}^{1} d \xi \xi^{n-1} H_{a, d}(\xi)=\frac{M_{2,3}^{n}}{n^{2}}, \\
\int_{0}^{1} d \xi \xi^{n-1} G_{a, d}(\xi)=\frac{M_{2,3}^{n}}{n}, \\
\int_{0}^{1} d \xi \xi^{n} F_{a, d}(\xi)=M_{2,3}^{n} .
\end{array}
$$

The result is consistent with the one obtained for the case of a polarized nucleon target in Ref. [21]. For phenomenological analysis, the experimental data will be used for the lefthand sides of Eqs. (3.16) and (3.17) which should be compared with the right-hand sides, the QCD predictions.

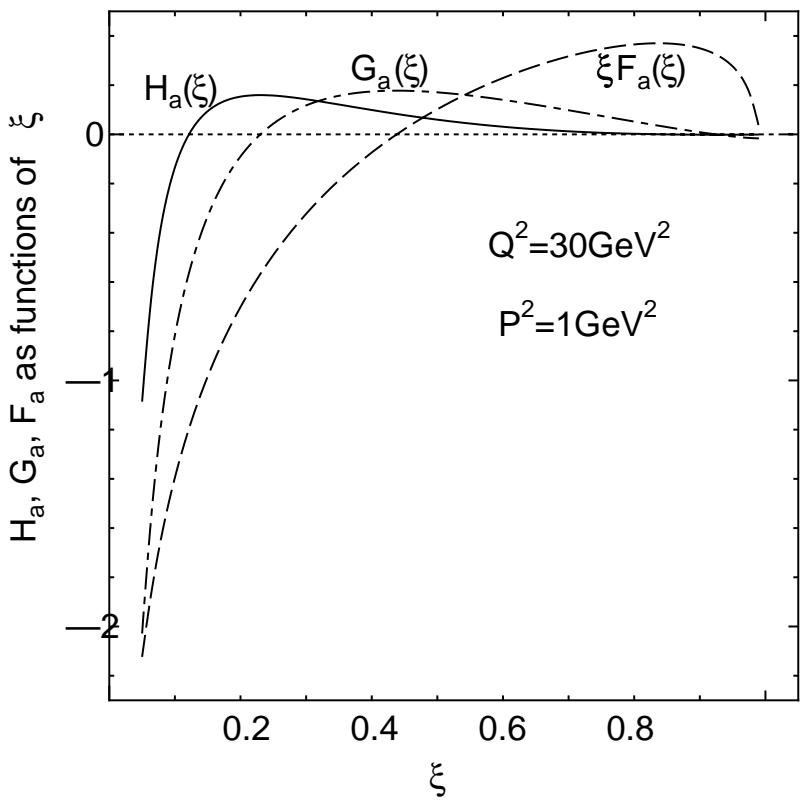

FIG. 3. The functions $H_{a}(\xi)$ (solid curve), $G_{a}(\xi)$ (dash-dotted curve), and $\xi F_{a}(\xi)$ (dashed curve) obtained by the inverse Mellin transform of the weighted moments of $M_{2}^{n}$ for twist-2 operators, Eqs. (4.1), (4.2), and (4.3).

\section{NUMERICAL ANALYSIS}

Let us perform a numerical analysis for the target mass effects in $g_{1}^{\gamma}$ and $g_{2}^{\gamma}$.

\section{A. $g_{1}^{\gamma}\left(x, Q^{2}, P^{2}\right)$ derived from $H_{a, d}(\xi), G_{a, d}(\xi)$, and $F_{a, d}(\xi)$}

We first compute the functions, $H_{a, d}(\xi), G_{a, d}(\xi)$, and $F_{a, d}(\xi)$, inverting the Nachtmann moments $M_{2}^{n}$ and $M_{3}^{n}$,

$$
\begin{aligned}
& H_{a, d}(\xi)=\frac{1}{2 \pi i} \int_{c-i \infty}^{c+i \infty} d n \xi^{-n} \frac{M_{2,3}^{n}}{n^{2}}, \\
& G_{a, d}(\xi)=\frac{1}{2 \pi i} \int_{c-i \infty}^{c+i \infty} d n \xi^{-n} \frac{M_{2,3}^{n}}{n},
\end{aligned}
$$

$$
\xi F_{a, d}(\xi)=\frac{1}{2 \pi i} \int_{c-i \infty}^{c+i \infty} d n \xi^{-n} M_{2,3}^{n}
$$

We use the QCD result for $M_{2}^{n}\left(=\Sigma_{i} a_{(2) i}^{\gamma, n} E_{(2) i}^{n}\right)$, which has been calculated up to NLO and given in Eq. (3.16) of the first article of Ref. [13]. As for $M_{3}^{n}\left(=\Sigma_{i} a_{(3) i}^{\gamma, n} E_{(3) i}^{n}\right)$, on the other hand, we adopt the pure QED result, Eq. (3.22) of Ref. [15], with the factor $(n-1) / n$ taken out. The QCD calculation of $M_{3}^{n}$ even in LO has not been accomplished yet. The evaluation of the twist-3 part $M_{3}^{n}$ in QCD is feasible when $n$ is a small number. But as $n$ gets larger it becomes a more and more difficult task due to the increase of the number of participating operators and the mixing among these operators [24].

We have plotted the twist-2 contributions, $H_{a}(\xi), G_{a}(\xi)$, and $\xi F_{a}(\xi)$ as functions of $\xi$ in Fig. 3, and the twist-3 contributions, $H_{d}(\xi), G_{d}(\xi)$, and $\xi F_{d}(\xi)$ in Fig. 4 , for the case 


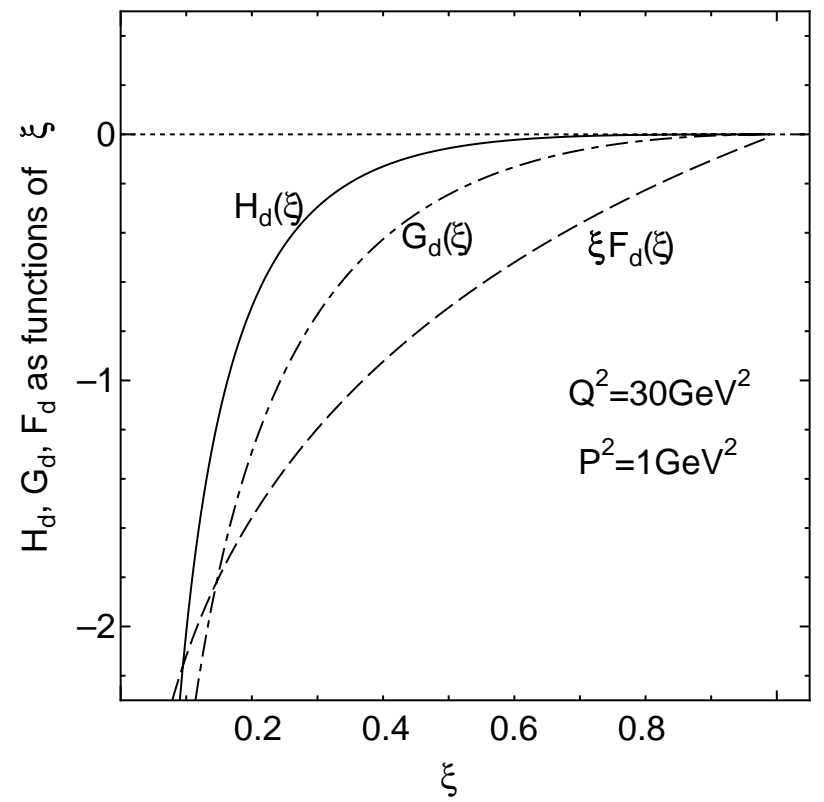

FIG. 4. The functions $H_{d}(\xi)$ (solid curve), $G_{d}(\xi)$ (dash-dotted curve), and $\xi F_{d}(\xi)$ (dashed curve) obtained by the inverse Mellin transform of the weighted moments of $M_{3}^{n}$ for twist-3 operators, Eqs. (4.1), (4.2), and (4.3).

of $Q^{2}=30 \mathrm{GeV}^{2}$ and $P^{2}=1 \mathrm{GeV}^{2}$. We take $\Lambda=0.2 \mathrm{GeV}$ for the QCD parameter and $N_{f}=3$ for the number of active quark flavors throughout our numerical analysis. We see that all the functions $H_{a, d}(\xi), G_{a, d}(\xi)$, and $F_{a, d}(\xi)$ vanish as $\xi$ $\rightarrow 1$. The behavior of a function near $\xi=1$ is governed by its moments at large $n$. The LO QCD result for $M_{2}^{n}$ gives $M_{2}^{n}$ $\sim 1 /(n \ln n)$ for large $n$, which determines the dominant behaviors of the functions near $\xi=1$, and thus we expect that

$$
\begin{aligned}
\xi F_{a}(\xi) & \sim-\frac{1}{\ln (1-\xi)}, \quad G_{a}(\xi) \sim \frac{\ln \xi}{\ln (1-\xi)}, \\
H_{a}(\xi) & \sim-\frac{(\ln \xi)^{2}}{\ln (1-\xi)} .
\end{aligned}
$$

For the twist-3 part, $M_{3}^{n}$, the pure QED result tells that $M_{3}^{n}$ $\sim-1 / n^{2}$ at large $n$. So we get near $\xi=1$,

$$
\xi F_{d}(\xi) \sim \ln \xi, \quad G_{d}(\xi) \sim-(\ln \xi)^{2}, \quad H_{d}(\xi) \sim(\ln \xi)^{3} .
$$

The behaviors of $H_{a, d}(\xi), G_{a, d}(\xi)$, and $F_{a, d}(\xi)$ as $\xi \rightarrow 1$ in Figs. 3 and 4 are indeed just what we have expected. The functions $H_{a, d}(\xi), G_{a, d}(\xi)$, and $F_{a, d}(\xi)$ for the case of $Q^{2}$ $=10 \mathrm{GeV}^{2}$ and $P^{2}=1 \mathrm{GeV}^{2}$ show the similar behaviors.

Putting these results into the formula (3.11), we obtain $g_{1}^{\gamma}\left(x, Q^{2}, P^{2}\right)$ with TME as a function of $x$, which is shown (solid curve) in Fig. 5 for $Q^{2}=30 \mathrm{GeV}^{2}$ with $P^{2}=1 \mathrm{GeV}^{2}$ and in Fig. 6 for $Q^{2}=10 \mathrm{GeV}^{2}$ with $P^{2}=1 \mathrm{GeV}^{2}$. The vertical axis is in units of $3 N_{f}\left\langle e^{4}\right\rangle(\alpha / \pi) \ln \left(Q^{2} / P^{2}\right)$, where $\alpha$ $=e^{2} / 4 \pi$, the QED coupling constant, and $\left\langle e^{4}\right\rangle=\Sigma_{i=1}^{N_{f}} e_{i}^{4} / N_{f}$ with $e_{i}$ being the electric charge of the $i$ th flavor quark. Also plotted are $\left.g_{1}^{\gamma}\left(x, Q^{2}, P^{2}\right)\right|_{0}$ without TME (dashed curve) [see

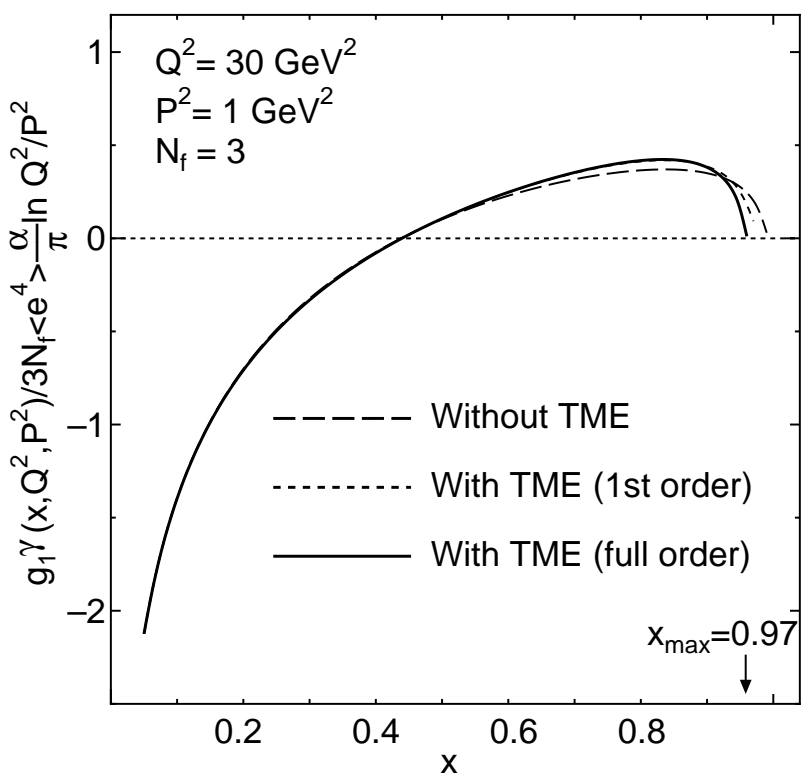

FIG. 5. The graphs of $g_{1}^{\gamma}\left(x, Q^{2}, P^{2}\right)$ with full TME [Eq. (3.11), solid curve] with the first order TME (short-dashed curve) and without TME (dashed curve) in units of $3 N_{f}\left\langle e^{4}\right\rangle(\alpha / \pi) \ln \left(Q^{2} / P^{2}\right)$ for $Q^{2}=30 \mathrm{GeV}^{2}$ and $P^{2}=1 \mathrm{GeV}^{2}$ with $\Lambda=0.2 \mathrm{GeV}, N_{f}=3$.

Eq. (3.13)] and the one with TME included up to the first order in $P^{2} / Q^{2}$ (short-dashed curve), which is obtained by the inverse Mellin transform of the right-hand side of Eq. (3.16). We observe that the target mass effects appear between intermediate $x$ and $x_{\max }$, and that the effects become sizable when the ratio $Q^{2} / P^{2}$ is reduced (see Fig. 6). The distinction between the behaviors of $g_{1}^{\gamma}$ with and without TME is remarkable near $x_{\max }$. We get $x_{\max } \approx 0.97$ for $Q^{2}$ $=30 \mathrm{GeV}^{2}$ with $P^{2}=1 \mathrm{GeV}^{2}$ and $x_{\max } \approx 0.91$ for $Q^{2}$ $=10 \mathrm{GeV}^{2}$ with $P^{2}=1 \mathrm{GeV}^{2}$. The graphs of $g_{1}^{\gamma}$ with TME

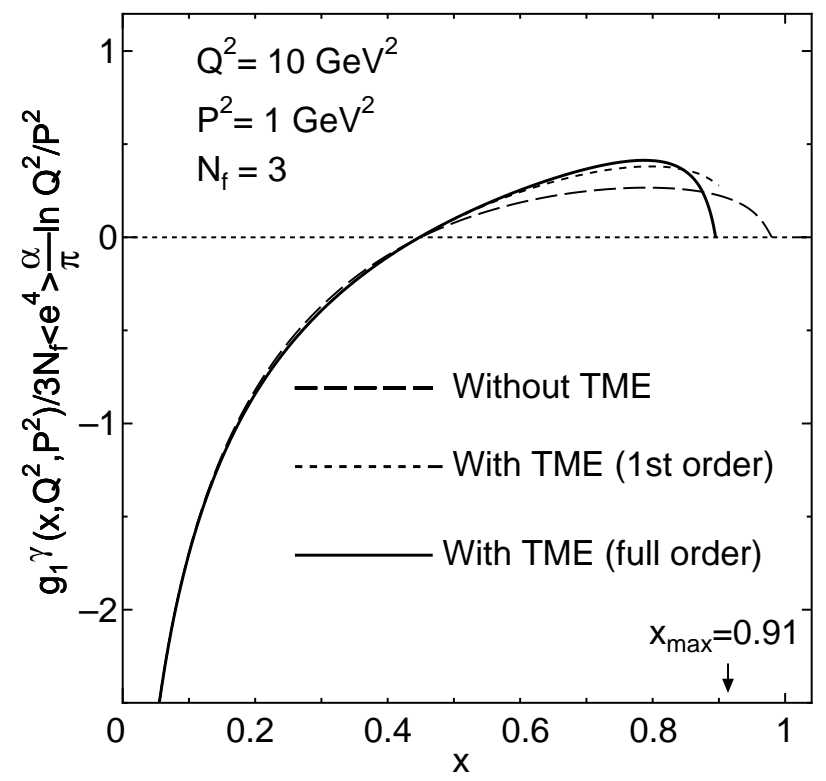

FIG. 6. The graphs of $g_{1}^{\gamma}\left(x, Q^{2}, P^{2}\right)$ for $Q^{2}=10 \mathrm{GeV}^{2}$ and $P^{2}$ $=1 \mathrm{GeV}^{2}$ with $\Lambda=0.2 \mathrm{GeV}, N_{f}=3$. 
vanish at $x_{\max }$ as they should. But those graphs without TME or with TME partially included remain finite. In the small $x$ region the target mass effects are almost negligible. We also note that the graph with the first order corrections in $P^{2} / Q^{2}$ is a good approximation to the full-order result except around $x_{\max }$.

\section{B. The first moment sum rule of $g_{1}^{\gamma}\left(x, Q^{2}, P^{2}\right)$ and target mass effects}

When the target mass corrections are not taken into account, the polarized virtual photon structure function $g_{1}^{\gamma}\left(x, Q^{2}, P^{2}\right)$ satisfies the following sum rule $[7,13]$ :

$$
\left.\Gamma_{1}^{\gamma} \equiv \int_{0}^{1} d x g_{1}^{\gamma}\left(x, Q^{2}, P^{2}\right)\right|_{0}=-\frac{3 \alpha}{\pi} \sum_{i=1}^{N_{f}} e_{i}^{4}+O\left(\alpha_{s}\right) .
$$

The right-hand side corresponds to the twist- 2 contribution, $M_{2}^{n=1}\left(=\Sigma_{i} a_{(2) i}^{\gamma, n=1} E_{(2) i}^{n=1}\right)$, and actually the first term is the consequence of the QED axial anomaly. Now it will be interesting to see how this sum rule is modified when TME are included.

From Eq. (2.17) we easily see that once the target mass corrections are taken into account, the above sum rule is modified to the first Nachtmann moment, which reads

$$
\begin{aligned}
& \frac{1}{9} \int_{0}^{x_{\max }} d x \frac{\xi^{2}}{x^{2}}\left[5+4 \sqrt{1-\frac{4 P^{2} x^{2}}{Q^{2}}}\right] g_{1}^{\gamma}\left(x, Q^{2}, P^{2}\right) \\
& +\frac{4}{3} \int_{0}^{x_{\max }} d x \frac{\xi^{2}}{x^{2}} \frac{P^{2} x^{2}}{Q^{2}} g_{2}^{\gamma}\left(x, Q^{2}, P^{2}\right) \\
& =-\frac{3 \alpha}{\pi} \sum_{i=1}^{N_{f}} e_{i}^{4}+O\left(\alpha_{s}\right) .
\end{aligned}
$$

Phenomenologically it would be appropriate to express the first moment of $g_{1}^{\gamma}\left(x, Q^{2}, P^{2}\right)$ itself in terms of $M_{2}^{n}$ and $M_{3}^{n}$, which are calculable by perturbative QCD. Setting $n=1$ in Eq. (3.16), we obtain to the first order in $P^{2} / Q^{2}$,

$$
\begin{aligned}
\int_{0}^{x_{\max }} & d x g_{1}^{\gamma}\left(x, Q^{2}, P^{2}\right) \\
\quad= & M_{2}^{n=1}-\left\{\frac{2}{9} M_{2}^{n=3}+\frac{8}{9} M_{3}^{n=3}\right\} \frac{P^{2}}{Q^{2}}+O\left(\left(P^{2} / Q^{2}\right)^{2}\right)
\end{aligned}
$$

where $M_{2}^{n=1}=-(3 \alpha / \pi) \Sigma_{i=1}^{N_{f}} e_{i}^{4}$. Thus the target mass corrections $\Delta \Gamma_{1}^{\gamma}$ to the first moment of $g_{1}^{\gamma}$, i.e., the difference between the left-hand sides of Eqs. (4.8) and (4.6) is given, to the first order in $P^{2} / Q^{2}$, by

$$
\Delta \Gamma_{1}^{\gamma}=-\left\{\frac{2}{9} M_{2}^{n=3}+\frac{8}{9} M_{3}^{n=3}\right\} \frac{P^{2}}{Q^{2}} .
$$

Up to this order in $P^{2} / Q^{2}$ we only need to know the reduced matrix elements and coefficient functions for $n=3$. Using the NLO result for $M_{2}^{n=3}$ in QCD, given in Eq. (3.16) of Ref. [13], we obtain

$$
\begin{aligned}
& M_{2}^{n=3} / \frac{\alpha}{\pi}=0.163(0.0601) \text { for } \\
& Q^{2}=30 \mathrm{GeV}^{2}\left(10 \mathrm{GeV}^{2}\right), \quad P^{2}=1 \mathrm{GeV}^{2} .
\end{aligned}
$$

As for the twist- 3 contribution $M_{3}^{n=3}$, the LO result in QCD is available. Taking the results in Eqs. (4.30)-(4.36) of Ref. [15], we get

$$
\begin{aligned}
& M_{3}^{n=3} / \frac{\alpha}{\pi}=-0.130(-0.0942) \text { for } \\
& Q^{2}=30 \mathrm{GeV}^{2}\left(10 \mathrm{GeV}^{2}\right), \quad P^{2}=1 \mathrm{GeV}^{2} .
\end{aligned}
$$

With these numerical values we find

$$
\begin{aligned}
& \Delta \Gamma_{1}^{\gamma} / M_{2}^{n=1}=-0.00395(-0.0106) \text { for } \\
& Q^{2}=30 \mathrm{GeV}^{2}\left(10 \mathrm{GeV}^{2}\right), \quad P^{2}=1 \mathrm{GeV}^{2}
\end{aligned}
$$

The target mass corrections to the first moment sum rule of $g_{1}^{\gamma}$ amount to $0.40 \%(1.1 \%)$ for $Q^{2}=30 \mathrm{GeV}^{2}\left(10 \mathrm{GeV}^{2}\right)$ with $P^{2}=1 \mathrm{GeV}^{2}$, which are negligibly small. Even for the latter case, $Q^{2}=10 \mathrm{GeV}^{2}$ and $P^{2}=1 \mathrm{GeV}^{2}$, the corrections are, at most, of order of $1 \%$. We see from Eqs. (4.10) and (4.11) that the twist-2 and twist-3 contributions $M_{2}^{n=3}$ and $M_{3}^{n=3}$ for $n=3$ are almost the same in magnitude but have the opposite signs. This leads to the smallness of target mass corrections to the first moment sum rule of $g_{1}^{\gamma}$.

\section{C. $g_{2}^{\gamma}\left(x, Q^{2}, P^{2}\right)$ and the target mass effects}

We obtain the graph of $g_{2}^{\gamma}\left(x, Q^{2}, P^{2}\right)$ with TME by inserting the functions $H_{a, d}(\xi), G_{a, d}(\xi)$, and $F_{a, d}(\xi)$ derived from Eqs. (4.1)-(4.3) into Eq. (3.12). Again we have used the pure QED result for $M_{3}^{n}$, Eq. (3.22) of Ref. [15], since the QCD result for $M_{3}^{n}$ with $n>3$ is not available. In Fig. 7 we have plotted $g_{2}^{\gamma}\left(x, Q^{2}, P^{2}\right)$ with TME (solid curve) in units of $3 N_{f}\left\langle e^{4}\right\rangle(\alpha / \pi) \ln \left(Q^{2} / P^{2}\right)$ for $Q^{2}=30 \mathrm{GeV}^{2}$ and $P^{2}$ $=1 \mathrm{GeV}^{2}$. Also shown in Fig. 7 is the box-diagram contribution to $g_{2}^{\gamma}$ (dashed curve) for an example without TME, the expression of which is given by [15]

$$
\begin{aligned}
g_{2}^{\gamma(\text { Box })}\left(x, Q^{2}, P^{2}\right)= & \frac{3 \alpha}{\pi} N_{f}\left\langle e^{4}\right\rangle\left[-(2 x-1) \ln \frac{Q^{2}}{P^{2}}\right. \\
& +2(2 x-1) \ln x+6 x-4] .
\end{aligned}
$$

The graph of $g_{2}^{\gamma}$ with TME vanishes at $x_{\max }$, but not the one without TME.

In a certain limit the analysis of $M_{3}^{n}$ in QCD becomes tractable. The contribution to $M_{3}^{n}$ is made up of two components; the flavor singlet and nonsinglet. In an approximation of neglecting terms of order $O\left(1 / N_{c}^{2}\right)$ we are able to calculate $M_{3}^{n(\mathrm{NS})}$, the flavor nonsinglet contribution to $M_{3}^{n}$, for arbitrary $n$ in QCD since in this limit the problem of operator mixing can be evaded [25]. In fact, we have computed $M_{3}^{n \text { (NS) }}$ in LO QCD for the large $N_{c}$ limit, which is given in 


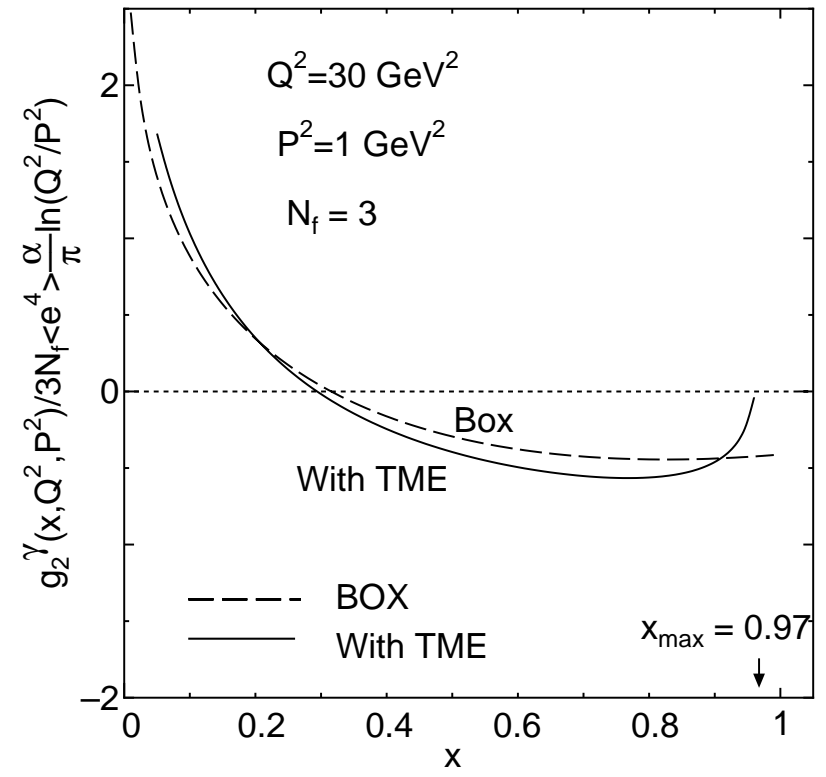

FIG. 7. The graph of $g_{2}^{\gamma}\left(x, Q^{2}, P^{2}\right)$ with TME [Eq. (3.12), solid curve] and the box-diagram contribution to $g_{2}^{\gamma}$ (dashed curve) in units of $3 N_{f}\left\langle e^{4}\right\rangle(\alpha / \pi) \ln \left(Q^{2} / P^{2}\right)$ for $Q^{2}=30 \mathrm{GeV}^{2}$ and $P^{2}$ $=1 \mathrm{GeV}^{2}$ with $\Lambda=0.2 \mathrm{GeV}, N_{f}=3$.

Eq. (4.40) of Ref. [15]. Using these $M_{3}^{n \text { (NS) }}$ we perform the inverse Mellin transform of Eqs. (4.1)-(4.3) to obtain the flavor nonsinglet contributions, $H_{d}^{\mathrm{NS}}(\xi), G_{d}^{\mathrm{NS}}(\xi)$, and $F_{d}^{\mathrm{NS}}(\xi)$. Then putting these functions into the formula (3.12) and setting the twist-2 contributions to zero, i.e., $H_{a}(\xi)$ $=G_{a}(\xi)=F_{a}(\xi)=0$, we obtain $\bar{g}_{2}^{\gamma(\mathrm{NS})}\left(x, Q^{2}, P^{2}\right)$, the twist-3 contribution to the flavor nonsinglet part of $g_{2}^{\gamma}$, including TME.

In Fig. 8 we have plotted $\bar{g}_{2}^{\gamma(\mathrm{NS})}\left(x, Q^{2}, P^{2}\right)$ with TME (solid curve) in units of $3 N_{f}\left(\left\langle e^{4}\right\rangle-\left\langle e^{2}\right\rangle^{2}\right)(\alpha / \pi) \ln \left(Q^{2} / P^{2}\right)$ for $Q^{2}=30 \mathrm{GeV}^{2}$ and $P^{2}=1 \mathrm{GeV}^{2}$, where $\left(\left\langle e^{4}\right\rangle-\left\langle e^{2}\right\rangle^{2}\right)$ is a charge factor for the flavor nonsinglet component with $\left\langle e^{2}\right\rangle=\sum_{i=1}^{N_{f}} e_{i}^{2} / N_{f}$. Also plotted are the graphs of $\bar{g}_{2}^{\gamma(\mathrm{NS})}$ without TME (short-dashed curve) and the box-diagram contribution to $\bar{g}_{2}^{\gamma(\mathrm{NS})}$ (dashed curve) which is obtained by subtracting the twist-2 contribution [27] from Eq. (4.13) and is given by [15]

$$
\begin{aligned}
\bar{g}_{2}^{\gamma(\mathrm{NS}, \mathrm{Box})}= & \frac{3 \alpha}{\pi} N_{f}\left(\left\langle e^{4}\right\rangle-\left\langle e^{2}\right\rangle^{2}\right)\left[(2 x-2-\ln x) \ln \frac{Q^{2}}{P^{2}}\right. \\
& \left.-2(2 x-1) \ln x+2(x-1)+\ln ^{2} x\right] .
\end{aligned}
$$

We observe that target mass corrections in the twist-3 part are negligibly small. This is inferred from the fact that target mass effects appear at large $\xi(\operatorname{large} x)$ and the twist-3 contributions $\xi F_{d}(\xi), G_{d}(\xi)$, and $H_{d}(\xi)$ vanish as $\ln \xi$, $-(\ln \xi)^{2}$, and $(\ln \xi)^{3}$, respectively, for $\xi \rightarrow 1$. Another case for $Q^{2}=10 \mathrm{GeV}^{2}$ with $P^{2}=1 \mathrm{GeV}^{2}$ is shown in Fig. 9, where we see that target mass effects become slightly larger than the case for $Q^{2}=30 \mathrm{GeV}^{2}$, in particular, in the region near $x_{\max }$.

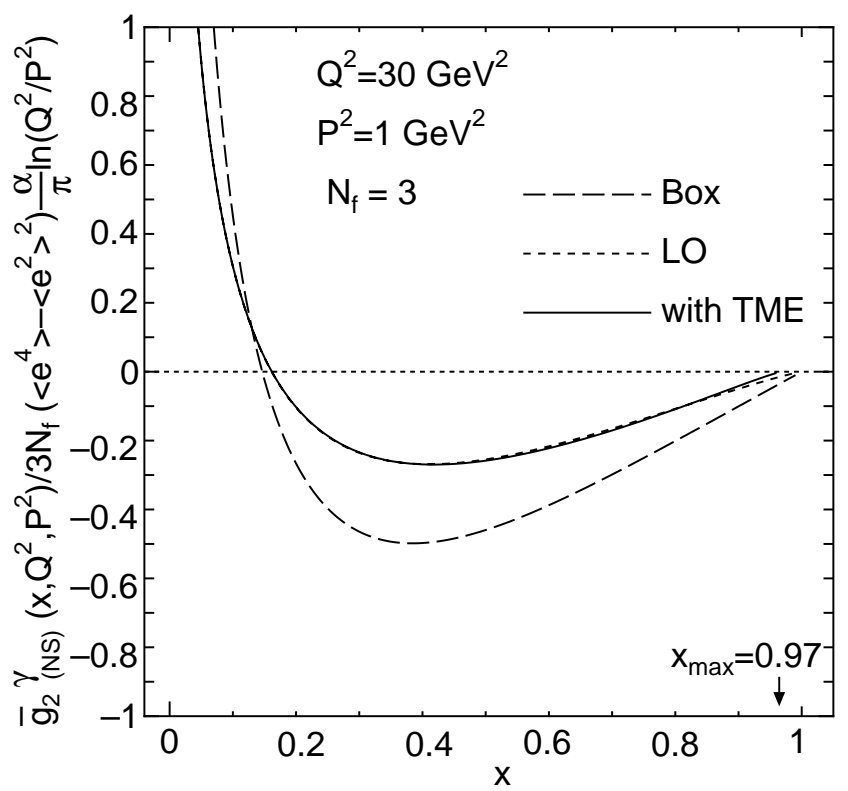

FIG. 8. The graphs of $\bar{g}_{2}^{\gamma(\mathrm{NS})}\left(x, Q^{2}, P^{2}\right)$, the twist-3 contribution to the flavor non-singlet part of $g_{2}^{\gamma}$, in units of $3 N_{f}\left(\left\langle e^{4}\right\rangle\right.$ $\left.-\left\langle e^{2}\right\rangle^{2}\right)(\alpha / \pi) \ln \left(Q^{2} / P^{2}\right)$ for $Q^{2}=30 \mathrm{GeV}^{2}$ and $P^{2}=1 \mathrm{GeV}^{2}$ with $\Lambda=0.2 \mathrm{GeV}, N_{f}=3$. The twist-3 effects are evaluated in LO QCD for the large $N_{c}$ limit. The solid and short-dashed curves show the results with TME and without TME, respectively. We have also shown the box-diagram contribution to $\bar{g}_{2}^{\gamma(\mathrm{NS})}$ (dashed curve) for comparison.

\section{Burkhardt-Cottingham sum rule}

Just as the spin-dependent nucleon structure function $g_{2}^{\text {nucl }}$, the polarized virtual photon structure function $g_{2}^{\gamma}\left(x, Q^{2}, P^{2}\right)$ satisfies the Burkhardt-Cottingham (BC) sum rule [26]

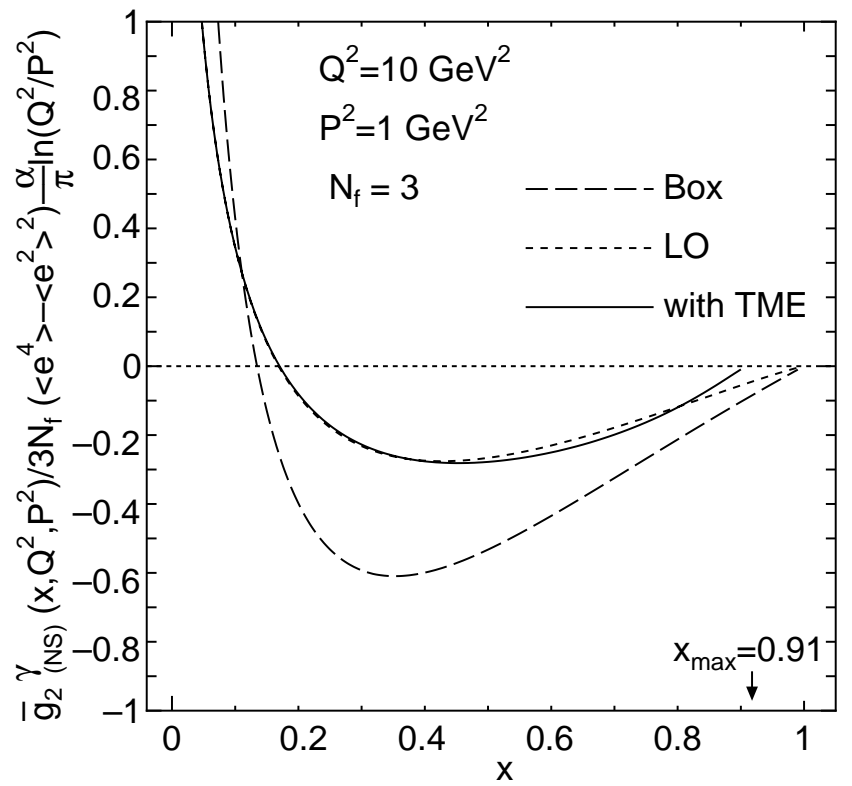

FIG. 9. The graphs of $\bar{g}_{2}^{\gamma(\mathrm{NS})}\left(x, Q^{2}, P^{2}\right)$ for $Q^{2}=10 \mathrm{GeV}^{2}$ and $P^{2}=1 \mathrm{GeV}^{2}$ with $\Lambda=0.2 \mathrm{GeV}, N_{f}=3$. 


$$
\left.\int_{0}^{1} d x g_{2}^{\gamma}\left(x, Q^{2}, P^{2}\right)\right|_{0}=0
$$

We put the subscript 0 to emphasize that this is the statement when the target mass corrections are not included. When the TME are included, Eq. (3.17) shows that the BC sum rule still holds up to the first order in $P^{2} / Q^{2}$. Actually we take the $x$ moments of $g_{2}^{\gamma}$ whose expression is given in Eq. (3.12). Using the relations in Eq. (3.10) and by partial integration with the boundary conditions $H_{a, d}(\xi=1)=G_{a, d}(\xi=1)=0$, we obtain

$$
\begin{aligned}
\int_{0}^{x_{\max }} & d x x^{n-1} g_{2}^{\gamma}\left(x, Q^{2}, P^{2}\right) \\
= & n(n-1) \int_{0}^{1} d \xi \frac{\xi^{n-1}}{\left(1+\kappa \xi^{2}\right)^{n+1}}\left[-H_{a}(\xi)\right. \\
& \left.+\left(1+\kappa \xi^{2}\right) H_{d}(\xi)\right] .
\end{aligned}
$$

Taking $n=1$, we arrive at

$$
\int_{0}^{x_{\max }} d x g_{2}^{\gamma}\left(x, Q^{2}, P^{2}\right)=0
$$

which shows the BC sum rule is free from the target mass effects. Note that the upper limit of integration has changed from 1 to $x_{\max }$. A similar expression to the one in Eq. (4.16) has been obtained by Piccione and Ridolfi [21] for the moments of the nucleon structure function $g_{2}^{\text {nucl }}$ when the target mass corrections are included.

\section{CONCLUSION}

In this paper we have investigated the target mass effects in the polarized virtual photon structure functions $g_{1}^{\gamma}\left(x, Q^{2}, P^{2}\right), g_{2}^{\gamma}\left(x, Q^{2}, P^{2}\right)$ which can be measured in the future experiments of the polarized version of the $e p$ or $e^{+} e^{-}$colliders.

We based our argument on the framework of the OPE and the $\mathrm{O}(4)$ expansion, taking into account the trace terms of the operators of the definite spin. This amounts to the use of the expansion of the amplitudes in terms of the Gegenbauer polynomials and their orthogonality relations to extract the contributions with the definite spin, given as the Nachtmann moments. The evaluation of the "kinematical" target mass effects is important to extract the "dynamical" higher-twist effects which would also exist in the power corrections in $P^{2} / Q^{2}$.

We have derived the expressions for $g_{1}^{\gamma}\left(x, Q^{2}, P^{2}\right)$ and $g_{2}^{\gamma}\left(x, Q^{2}, P^{2}\right)$ in closed form by inverting the Nachtmann moments for the twist- 2 and twist- 3 operators. The characteristic feature for the photon target compared to the nucleon case is the presence of the maximal value $x_{\max }(<1)$ of the Bjorken variable $x$, while $\xi\left(x_{\max }\right)=1$. Hence we do not encounter the similar problem due to the kinematical relation $\xi\left(x_{\max }\right)<1$ with $x_{\max }=1$ in the case of the nucleon.

Our numerical analysis shows that the target mass effects appear at large $x$ and become sizable near $x_{\max }(=1 /[1$
$\left.\left.+\left(P^{2} / Q^{2}\right)\right]\right)$, as the ratio $P^{2} / Q^{2}$ increases. The structure functions evaluated by inverting the Nachtmann moments in fact vanish at $x=x_{\max }$. We have also examined the target mass effects for the first-moment sum rules of $g_{1}^{\gamma}$ and $g_{2}^{\gamma}$. For the kinematic region we consider, the corrections to the first moment of $g_{1}^{\gamma}$ turn out to be negligibly small. The first moment of $g_{2}^{\gamma}$ leads to the Burkhardt-Cottingham sum rule, where the upper limit of integration becomes $x_{\max }$. More thorough QCD analysis, including the flavor-singlet part of $g_{2}^{\gamma}$, is now under investigation.

\section{ACKNOWLEDGMENTS}

This work is partially supported by the Grant-in-Aid for Scientific Research from the Ministry of Education, Culture, Sports, Science and Technology, No.(C)(2)-15540266.

\section{APPENDIX A: GEGENBAUER POLYNOMIALS}

In this appendix we present main formulas of Gegenbauer polynomials which are used in this paper. The Gegenbauer polynomials $C_{n}^{(\nu)}(\eta)$ are defined through the generating function given by $[28,29]$

$$
\left(1-2 \eta t+t^{2}\right)^{-\nu}=\sum_{n=0}^{\infty} C_{n}^{(\nu)}(\eta) t^{n}
$$

In terms of hypergeometric functions $F(\alpha, \beta, \gamma ; z), C_{n}^{(\nu)}(\eta)$ is expressed as

$$
\begin{aligned}
C_{n}^{(\nu)}(\eta) & =\frac{2^{n} \Gamma(n+\nu)}{n ! \Gamma(\nu)} F\left(-\frac{n}{2}, \frac{1-n}{2}, 1-n-\nu ; \frac{1}{\eta^{2}}\right) \\
& =\frac{1}{\Gamma(\nu)} \sum_{j=0}^{n / 2} \frac{(-1)^{j} \Gamma(\nu+n-j)}{j !(n-2 j) !}(2 \eta)^{n-2 j},
\end{aligned}
$$

from which we obtain, for example,

$$
\begin{aligned}
C_{n}^{(1)}(\eta) & =\sum_{j=0}^{n / 2} \frac{(-1)^{j}}{j !} \frac{(n-j) !}{(n-2 j) !}(2 \eta)^{n-2 j}, \\
C_{n-1}^{(2)}(\eta) & =\sum_{j=0}^{(n-1) / 2} \frac{(-1)^{j}}{j !} \frac{(n-j) !}{(n-2 j-1) !}(2 \eta)^{n-2 j-1} .
\end{aligned}
$$

\section{Recursion formulas}

$$
\begin{aligned}
n C_{n}^{(\nu)}(\eta)= & 2 \nu\left[\eta C_{n-1}^{(\nu+1)}(\eta)-C_{n-2}^{(\nu+1)}(\eta)\right], \\
(n+2 \nu) C_{n}^{(\nu)}(\eta)= & 2 \nu\left[C_{n}^{(\nu+1)}(\eta)-\eta C_{n-1}^{(\nu+1)}(\eta)\right], \\
(n+2) C_{n+2}^{(\nu)}(\eta)= & 2(n+\nu+1) \eta C_{n+1}^{(\nu)}(\eta) \\
& -(n+2 \nu) C_{n}^{(\nu)}(\eta) .
\end{aligned}
$$


2. Orthogonality relations

$$
\begin{gathered}
\int_{-1}^{1}\left(1-\eta^{2}\right)^{\nu-(1 / 2)} C_{m}^{(\nu)}(\eta) C_{n}^{(\nu)}(\eta) d \eta \\
\quad=\frac{2 \pi}{2^{2 \nu}} \frac{\Gamma(n+2 \nu)}{(n+\nu) n ![\Gamma(\nu)]^{2}} \delta_{m n}
\end{gathered}
$$

In addition we need the following formula for the integral to project out the contributions of definite spin from the dispersion relations:

$$
\begin{aligned}
& \int_{-1}^{1} d \eta \eta^{m}\left(1-\eta^{2}\right)^{\nu-(1 / 2)} C_{n}^{(\nu)}(\eta) \frac{1}{\zeta-\eta} \\
& \quad=\frac{\pi}{2^{\nu-1}} \zeta^{m}\left(\zeta^{2}-1\right)^{(\nu-1) / 2}\left[\zeta-\left(\zeta^{2}-1\right)^{1 / 2}\right]^{n+\nu} \\
& \quad \times \frac{\Gamma(n+2 \nu)}{\Gamma(\nu) \Gamma(n+\nu+1)} \\
& \quad \times F\left(\nu, 1-\nu, n+\nu+1 ; \frac{-\zeta+\left(\zeta^{2}-1\right)^{1 / 2}}{2\left(\zeta^{2}-1\right)^{1 / 2}}\right) .
\end{aligned}
$$

In fact the factor $\left[\zeta-\left(\zeta^{2}-1\right)^{1 / 2}\right]^{n+\nu}$ gives $(-P / Q)^{n+\nu} \xi^{n+\nu}$, where $\xi$ is the so-called $\xi$-scaling variable given in Eq. (2.19).

\section{APPENDIX B: DERIVATION OF EQS. (2.11), (2.12)}

\section{Contraction formulas}

We give a table of contractions which are used for the derivation. First we introduce the most general rank- $n$ symmetric and traceless tensor, $\Pi^{\mu_{1} \cdots \mu_{n}}$, that can be formed with the momentum $p$ alone [17],

$$
\Pi^{\mu_{1} \cdots \mu_{n}}=\sum_{j=0}^{n / 2} \frac{(-1)^{j}}{2^{j}} \frac{(n-j) !}{n !} \underbrace{g \cdots g}_{j} \overbrace{p \cdots p}^{n-2 j}\left(p^{2}\right)^{j},
$$

where $g \cdots g$ stands for a product of $j$ metric tensors $g^{\mu_{l} \mu_{k}}$ with $2 j$ indices chosen among $\mu_{1}, \ldots, \mu_{n}$ in all possible ways. Then we easily find that the contraction of $\Pi^{\mu_{1} \cdots \mu_{n}}$ with $q_{\mu_{1}} \cdots q_{\mu_{n}}$ is expressed in terms of the Gegenbauer polynomial $C_{n}^{(1)}(\eta)$ given in Eq. (A3) $[16,18]$,

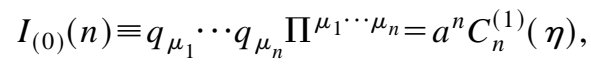

where

$$
a=-\frac{1}{2} P Q, \quad \eta=-\frac{p \cdot q}{P Q} .
$$

Next we differentiate both sides of Eq. (B2) with respect to $p^{\beta}$. Using the following formulas:

$$
\begin{aligned}
\frac{\partial a}{\partial p^{\beta}} & =a\left(\frac{-p_{\beta}}{P^{2}}\right), \quad \frac{\partial \eta}{\partial p^{\beta}}=\frac{q_{\beta}}{2 a}+\eta \frac{p_{\beta}}{P^{2}}, \\
\frac{d C_{n}^{(\nu)}(\nu)}{d \eta} & =2 \nu C_{n-1}^{(\nu+1)}(\eta),
\end{aligned}
$$

we find

$$
\begin{aligned}
n q_{\mu_{1}} & \cdots q_{\mu_{n}}\left\{\delta_{\beta}^{\mu_{1}} p^{\mu_{2} \cdots} p^{\left.\mu_{n-1}\right\}_{n}}\right. \\
& =\left\{-n a^{n} \frac{p_{\beta}}{P^{2}} C_{n}^{(1)}(\eta)+a^{n}\left[\frac{q_{\beta}}{2 a}+\eta \frac{p_{\beta}}{P^{2}}\right] 2 C_{n-1}^{(2)}(\eta)\right\},
\end{aligned}
$$

where $\left\{\delta_{\beta}^{\mu_{1}} p^{\mu_{2} \cdots} p^{\mu_{n-1}}\right\}_{n}$ is a tensor which is formed with one $\delta_{\beta}^{\mu_{i}}$ and $n-1$ momentum four-vectors $p$ and totally symmetric among indices $\mu_{1} \cdots \mu_{n}$. Moreover, it is traceless in the sense that $g_{\mu_{i} \mu_{j}}\left\{\delta_{\beta}^{\mu_{1}} p^{\mu_{2} \ldots} p^{\mu_{n-1}}\right\}_{n}=0$ for all pairs $i, j$. Now it is reminded that the polarized photon matrix elements are multiplied by the factor $\epsilon_{\rho \tau \alpha}{ }^{\beta}$. Thus the terms with $p_{\beta}$ in contractions do not contribute in the end. Also the terms with $q^{\sigma}$ which appear later on give null results when multiplied by $\epsilon_{\mu \nu \lambda \sigma}{ }^{\lambda}$ [see Eq. (2.10)]. So we obtain

$$
\begin{aligned}
I_{(1) \beta}(n)= & q_{\mu_{1}} \cdots q_{\mu_{n}}\left\{\delta_{\beta}^{\mu_{1}} p^{\mu_{2} \cdots} p^{\left.\mu_{n-1}\right\}_{n}}=\frac{1}{n} q_{\beta} a^{n-1} C_{n-1}^{(2)}(\eta)\right. \\
& +\left(\text { terms with } p_{\beta}\right) .
\end{aligned}
$$

Further, we differentiate both sides of Eq. (B6) with respect to $q_{\sigma}$. With

$$
\frac{\partial a}{\partial q_{\sigma}}=a\left(\frac{-q^{\sigma}}{Q^{2}}\right), \quad \frac{\partial \eta}{\partial q_{\sigma}}=\frac{p^{\sigma}}{2 a}+\eta \frac{q^{\sigma}}{Q^{2}},
$$

we obtain

$$
\begin{aligned}
I_{(2) \beta}^{\sigma}(n-1)= & q_{\mu_{1}} \cdots q_{\mu_{n-1}}\left\{\delta_{\beta}{ }^{\sigma} p^{\mu_{1} \cdots} p^{\left.\mu_{n-1}\right\}_{n}}=\frac{1}{n} \frac{\partial I_{(1) \beta}(n)}{\partial q_{\sigma}}\right. \\
= & \frac{1}{n^{2}}\left\{\delta_{\beta}{ }^{\sigma} a^{n-1} C_{n-1}^{(2)}(\eta)+q_{\beta} p^{\sigma} a^{n-2} 2 C_{n-2}^{(3)}(\eta)\right\} \\
& +\left(\text { terms with } p_{\beta} \text { or } q^{\sigma}\right) .
\end{aligned}
$$

Finally, both sides of Eq. (B2) are differentiated with respect to $q_{\sigma}$, and we get

$$
\begin{aligned}
I_{(3)}^{\sigma}(n-1) & =q_{\mu_{1}} \cdots q_{\mu_{n-1}} \Pi^{\sigma \mu_{1} \cdots \mu_{n-1}}=\frac{1}{n} \frac{\partial I_{(0)}(n)}{\partial q_{\sigma}} \\
& =\frac{1}{n}\left\{p^{\sigma} a^{n-1} C_{n-1}^{(2)}(\eta)\right\}+\left(\text { terms with } q^{\sigma}\right)
\end{aligned}
$$

The terms with $q^{\sigma}$ which appear in Eqs. (B8) and (B9) have been omitted. With Eqs. (B2), (B6), (B8), and (B9) at hand, we are now ready to derive Eqs. (2.11) and (2.12). 


\section{Derivation}

The tensor $\tilde{M}_{(2) \beta}^{\sigma \mu_{1} \cdots \mu_{n-1}}$, which corresponds to the traceless twist-2 operator $R_{(2) i}^{n}$, is formed one $\delta_{\beta}^{\sigma}$ (or $\delta_{\beta}^{\mu_{i}}$ ) and $n-1$ momentum four-vectors $p$ and is totally symmetric among indices $\sigma, \mu_{1} \cdots \mu_{n-1}$. Thus it is given by

$$
\tilde{M}_{(2) \beta}^{\sigma \mu_{1} \cdots \mu_{n-1}}=\left\{\delta_{\beta}^{\sigma} p^{\mu_{1} \cdots} p^{\mu_{n-1}}\right\}_{n} .
$$

For its contraction with $q_{\mu_{1}} \cdots q_{\mu_{n-1}}$, we find from Eq. (B8)

$$
\begin{aligned}
q_{\mu_{1}} \cdots & q_{\mu_{n-1}} \tilde{M}_{(2) \beta}^{\sigma \mu_{1} \cdots \mu_{n-1}} \\
= & I_{(2) \beta}^{\sigma}(n-1) \\
= & \frac{1}{n^{2}}\left\{\delta_{\beta}{ }^{\sigma} a^{n-1} C_{n-1}^{(2)}(\eta)+q_{\beta} p^{\sigma} a^{n-2} 2 C_{n-2}^{(3)}(\eta)\right\} \\
& \quad+\left(\text { terms with } p_{\beta} \text { or } q^{\sigma}\right) .
\end{aligned}
$$

The tensor $\tilde{M}_{(3) \beta}^{\sigma \mu_{1} \cdots \mu_{n-1}}$, which corresponds to the traceless twist-3 operator $R_{(3) i}^{n}$, is also formed one $\delta_{\beta}^{\sigma}$ (or $\delta_{\beta}^{\mu_{i}}$ ) and $n-1$ momentum four-vectors $p$. Its indices are totally symmetric among $\mu_{1} \cdots \mu_{n-1}$ but antisymmetric under $\sigma \leftrightarrow \mu_{i}$. The one which satisfies these requirements is [21]

$$
\begin{aligned}
\tilde{M}_{(3) \beta}^{\sigma \mu_{1} \cdots \mu_{n-1}=} & \frac{n-1}{n}\left\{\frac { n + 1 } { n } \left[\delta_{\beta}{ }^{\sigma} \Pi^{\mu_{1} \cdots \mu_{n-1}}-\frac{1}{(n-1)}\right.\right. \\
& \left.\times \sum_{l=1}^{n-1} \delta_{\beta}^{\mu_{l}} \Pi^{\sigma \mu_{1} \cdots\left(\mu_{l}\right) \cdots \mu_{n-1}}\right] \\
& +\frac{n-1}{n}\left[p^{\sigma} \tilde{M}_{(2) \beta}^{\mu_{1} \cdots \mu_{n-1}}-\frac{1}{(n-1)}\right. \\
& \left.\left.\times \sum_{l=1}^{n-1} p^{\mu_{l}} \tilde{M}_{(2) \beta}^{\sigma \mu_{1} \cdots\left(\mu_{l}\right) \cdots \mu_{n-1}}\right]\right\} .
\end{aligned}
$$

Using Eqs. (B2), (B6), (B8), (B9), we obtain

$$
\begin{aligned}
q_{\mu_{1}} \cdots q_{\mu_{n-1}} \tilde{M}_{(3) \beta}^{\sigma \mu_{1} \cdots \mu_{n-1}=} & \frac{n-1}{n}\left\{\frac{n+1}{n}\left[\delta_{\beta}{ }^{\sigma} I_{(0)}(n-1)-q_{\beta} I_{(3)}{ }^{\sigma}(n-2)\right]+\frac{n-1}{n}\left[p^{\sigma} I_{(1)}(n-1)-p \cdot q I_{(2)}{ }_{\beta}(n-2)\right]\right\} \\
= & \frac{1}{n^{2}} \delta_{\beta}{ }^{\sigma} a^{n-1}\left\{(n-1)(n+1) C_{n-1}^{(1)}(\eta)-2 \eta C_{n-2}^{(2)}(\eta)\right\}+\frac{1}{n^{2}} q_{\beta} p^{\sigma} a^{n-2}\left\{-2 C_{n-2}^{(2)}(\eta)\right. \\
& \left.-4 \eta C_{n-3}^{(3)}(\eta)\right\}+\left(\text { terms with } p_{\beta} \text { or } q^{\sigma}\right) \\
= & \frac{1}{n^{2}} \delta_{\beta}{ }^{\sigma} a^{n-1}\left\{(n-1) C_{n-1}^{(2)}(\eta)-(n+1) C_{n-3}^{(2)}(\eta)\right\}-\frac{1}{n^{2}} q_{\beta} p^{\sigma} a^{n-2} 2\left\{C_{n-2}^{(3)}(\eta)+C_{n-4}^{(3)}(\eta)\right\} \\
& +\left(\text { terms with } p_{\beta} \text { or } q^{\sigma}\right),
\end{aligned}
$$

where at the final stage the recursion relations in Eqs. (A5) and (A6) were used.

[1] M. Krawczyk, talk given at Photon 2000, edited by A. J. Finch, AIP Conf. Proc. 571 (AIP, Melville, NY, 2001), p. 3, and references therein.

[2] R. Nisius, talk given at Photon 2001, Proc. Photon 2001, hep-ex/0110078.

[3] M. Klasen, Rev. Mod. Phys. 74, 1221 (2002).

[4] I. Schienbein, Ann. Phys. (N.Y.) 301, 128 (2002).

[5] A. V. Efremov and O. V. Teryaev, JINR Report No. E2-88-287, Dubna, 1988; Phys. Lett. B 240, 200 (1990).

[6] S. D. Bass, Int. J. Mod. Phys. A 7, 6039 (1992).

[7] S. Narison, G. M. Shore, and G. Veneziano, Nucl. Phys. B391, 69 (1993); G. M. Shore and G. Veneziano, Mod. Phys. Lett. A 8, 373 (1993); Nucl. Phys. B381, 23 (1992).

[8] A. Freund and L. M. Sehgal, Phys. Lett. B 341, 90 (1994).

[9] S. D. Bass, S. J. Brodsky, and I. Schmidt, Phys. Lett. B 437, 424 (1998).

[10] K. Sasaki, Phys. Rev. D 22, 2143 (1980); Suppl. Prog. Theor. Phys. 77, 197 (1983).
[11] M. Stratmann and W. Vogelsang, Phys. Lett. B 386, 370 (1996).

[12] M. Glück, E. Reya, and C. Sieg, Phys. Lett. B 503, 285 (2001); Eur. Phys. J. C 20, 271 (2001).

[13] K. Sasaki and T. Uematsu, Phys. Rev. D 59, 114011 (1999); Phys. Lett. B 473, 309 (2000); Eur. Phys. J. C 20, 283 (2001).

[14] T. Uematsu and T. F. Walsh, Phys. Lett. 101B, 263 (1981); Nucl. Phys. B199, 93 (1982).

[15] H. Baba, K. Sasaki, and T. Uematsu, Phys. Rev. D 65, 114018 (2002).

[16] O. Nachtmann, Nucl. Phys. B63, 237 (1973); B78, 455 (1974).

[17] H. Georgi and H. Politzer, Phys. Rev. D 14, 1829 (1976).

[18] S. Wandzura, Nucl. Phys. B122, 412 (1977).

[19] S. Matsuda and T. Uematsu, Nucl. Phys. B168, 181 (1980).

[20] H. Kawamura and T. Uematsu, Phys. Lett. B 343, 346 (1995).

[21] A. Piccione and G. Ridolfi, Nucl. Phys. B513, 301 (1998).

[22] J. Blümlein and A. Tkabladze, Nucl. Phys. B553, 427 (1999). 
[23] A. De Rujula, H. Georgi, and H. Politzer, Ann. Phys. (N.Y.) 103, 315 (1977); Phys. Rev. D 15, 2495 (1977).

[24] E. V. Shuryak and A. I. Vainshtein, Nucl. Phys. B199, 451 (1982); B201, 141 (1982); A. P. Bukhvostov, E. A. Kuraev, and L. N. Lipatov, Sov. Phys. JETP 60, 22 (1984); P. G. Ratcliffe, Nucl. Phys. B264, 493 (1986); I. I. Balitsky and V. M. Braun, ibid. B311, 541 (1988); X. Ji and C. Chou, Phys. Rev. D 42, 3637 (1990); R. L. Jaffe, Comments Nucl. Part. Phys. 19, 239 (1990); R. L. Jaffe and X. Ji, Phys. Rev. D 43, 724 (1991); D. Müller, Phys. Lett. B 407, 314 (1997); V. M. Braun, G. P. Korchemsky, and A. N. Manashov, ibid. 476, 455 (2000); Nucl. Phys. B597, 370 (2001); B603, 69 (2001); J. Kodaira, T.
Uematsu, and Y. Yasui, Phys. Lett. B 344, 348 (1995); J. Kodaira, K. Tanaka, T. Uematsu, and Y. Yasui, ibid. 387, 855 (1996).

[25] A. Ali, V. M. Braun, and G. Hiller, Phys. Lett. B 266, 117 (1991); K. Sasaki, Phys. Rev. D 58, 094007 (1998).

[26] H. Burkhardt and W. N. Cottingham, Ann. Phys. (N.Y.) 56, 453 (1970).

[27] S. Wandzura and F. Wilczek, Phys. Lett. 72B, 195 (1977).

[28] A. Erdélyi et al., Higher Transcendental Functions, Bateman Manuscript Project (McGraw-Hill, New York, 1953), Vol. 1.

[29] I. S. Gradshteyn and I. M. Ryzhik, Tables of Integrals, Series, and Products (Academic Press, New York, 1965). 